\title{
Saddle-shaped solutions of bistable elliptic equations involving the half-Laplacian
}

\author{
ELEONORA CINTI
}

\begin{abstract}
We establish existence and qualitative properties of saddle-shaped solutions of the elliptic fractional equation $(-\Delta)^{1 / 2} u=f(u)$ in the whole space $\mathbb{R}^{2 m}$, where $f$ is of bistable type. These solutions are odd with respect to the Simons cone and even with respect to each coordinate.

More precisely, we prove the existence of a saddle-shaped solution in every even dimension $2 m$, as well as its monotonicity properties, asymptotic behaviour, and instability in dimensions $2 m=4$ and $2 m=6$.

These results are relevant in connection with the analog for fractional equations of a conjecture of De Giorgi on the 1-D symmetry of certain solutions. Saddle-shaped solutions are the simplest candidates, besides 1-D solutions, to be global minimizers in high dimensions, a property not yet established.
\end{abstract}

Mathematics Subject Classification (2010): 35J61 (primary); 35J20, 35B40, 35B08 (secondary).

\section{Introduction and results}

This paper concerns the study of saddle-shaped solutions of elliptic equations with fractional diffusion of the form

$$
(-\Delta)^{1 / 2} u=f(u) \text { in } \mathbb{R}^{n}
$$

where $n=2 m$ is an even integer and $f$ is of bistable type.

The fractional powers of the Laplacian are the infinitesimal generators of Lévy stable processes and appear in anomalous diffusion phenomena in plasmas, flame propagation, chemical reactions in liquids and population dynamics.

The author was supported by grants MTM2008-06349-C03-01, MTM2011-27739-C04-01 (Spain), 2009SGR345 (Catalunya) and partially supported by University of Bologna (Italy), funds for selected research topics.

Received July 12, 2011; accepted in revised form November 7, 2011. 
Our interest in saddle-shaped solutions originates from the following conjecture of De Giorgi. Consider the Allen-Cahn equation

$$
-\Delta u=u-u^{3} \quad \text { in } \mathbb{R}^{n},
$$

which models phase transitions. In 1978 De Giorgi conjectured that the level sets of every bounded solution of (1.2), which is monotone in one direction, must be hyperplanes, at least if $n \leq 8$. That is, such solutions depend only on one Euclidian variable.

The conjecture has been proven to be true in dimension $n=2$ by Ghoussoub and Gui [16] and in dimension $n=3$ by Ambrosio and Cabré [2]. For $4 \leq n \leq 8$, if $\partial_{x_{n}} u>0$, and assuming the additional condition

$$
\lim _{x_{n} \rightarrow \pm \infty} u\left(x^{\prime}, x_{n}\right)= \pm 1 \quad \text { for all } x^{\prime} \in \mathbb{R}^{n-1}
$$

it has been established by Savin [20]. Recently a counterexample to the conjecture for $n \geq 9$ has been found by del Pino, Kowalczyk and Wei [14].

For the fractional equation $(-\Delta)^{s} u=f(u)$ in $\mathbb{R}^{n}$ with $0<s<1$, the conjecture has been proven to be true when $n=2$ and $s=1 / 2$ by Cabré and SolàMorales [8], and when $n=2$ and for every $0<s<1$ by Cabré and Sire [6], and by Sire and Valdinoci [22]. In two recent papers [4,5], Cabré and the author prove the conjecture in dimension $n=3$ for every power $1 / 2 \leq s<1$.

Coming back to the classical Allen-Cahn equation, Savin [20], proved that if $n \leq 7$ then every global minimizer of the equation $-\Delta u=u-u^{3}$ in $\mathbb{R}^{n}$ is onedimensional. A natural question arises: is there a global minimizer in $\mathbb{R}^{8}$ which is not one-dimensional? Saddle-shaped solutions are the candidates to give a positive answer to this question, which is still an open problem.

Moreover, by a result of Jerison and Monneau [17], if one could prove that saddle-shaped solutions are global minimizers in $\mathbb{R}^{8}$, one would have a counterexample to the conjecture of De Giorgi in $\mathbb{R}^{9}$, in an alternative way to that of [14].

Saddle-shaped solutions are expected to have relevant variational properties due to a well known connection between nonlinear equations modeling phase transitions and the theory of minimal surfaces. This connection also motivated the conjecture of De Giorgi.

More precisely, the saddle-shaped solutions that we consider are even with respect to the coordinate axes and odd with respect to the Simons cone, which is defined as follows. For $n=2 m$ the Simons cone $\mathcal{C}$ is given by:

$$
\mathcal{C}=\left\{x \in \mathbb{R}^{2 m}: x_{1}^{2}+\ldots+x_{m}^{2}=x_{m+1}^{2}+\ldots+x_{2 m}^{2}\right\} \text {. }
$$

We recall that the Simons cone has zero mean curvature at every point $x \in \mathcal{C} \backslash\{0\}$, in every dimension $2 m \geq 2$. Moreover Bombieri, De Giorgi, and Giusti [3] proved that in dimensions $2 m \geq 8$ it is a minimizer of the area functional, that is, it is a minimal cone (in the variational sense). 
We define two new variables

$$
s=\sqrt{x_{1}^{2}+\cdots+x_{m}^{2}} \text { and } t=\sqrt{x_{m+1}^{2}+\cdots+x_{2 m}^{2}},
$$

for which the Simons cone becomes $\mathcal{C}=\{s=t\}$.

We now introduce the notion of saddle-shaped solution. These solutions depend only on $s$ and $t$, and are odd with respect to the Simons cone.

Definition 1.1. Let $u$ be a bounded solution of $(-\Delta)^{1 / 2} u=f(u)$ in $\mathbb{R}^{2 m}$, where $f \in C^{1}$ is odd. We say that $u: \mathbb{R}^{2 m} \rightarrow \mathbb{R}$ is a saddle-shaped (or simply saddle) solution if

(a) $u$ depends only on the variables $s$ and $t$. We write $u=u(s, t)$;

(b) $u>0$ for $s>t$;

(c) $u(s, t)=-u(t, s)$.

Remark 1.2. If $u$ is a saddle solution then, in particular, $u=0$ on the Simons cone $\mathcal{C}=\{s=t\}$. In other words, $\mathcal{C}$ is the zero level set of $u$.

Saddle solutions for the classical equation $-\Delta u=f(u)$ were first studied by Dang, Fife, and Peletier in [13] in dimension $2 m=2$ for $f$ odd, bistable and $f(u) / u$ decreasing for $u \in(0,1)$. They proved the existence and uniqueness of saddle-shaped solutions and established monotonicity properties and the asymptotic behaviour. The instability property of saddle solutions in dimension $2 m=2$ was studied by Schatzman [21]. In two recent works [10,11], Cabré and Terra proved the existence of saddle-shaped solutions for the equation $-\Delta u=f(u)$ in $\mathbb{R}^{2 m}$, where $f$ is of bistable type, in every even dimension $2 \mathrm{~m}$. Moreover they established some qualitative properties of these solutions, such as monotonicity properties, asymptotic behaviour, and also instability in dimensions $2 m=4$ and $2 m=6$.

In this work, we establish existence and qualitative properties of saddle-shaped solutions for the bistable fractional equation (1.1).

To study the nonlocal problem (1.1) we will realize it as a local problem in $\mathbb{R}_{+}^{n+1}$ with a nonlinear Neumann condition on $\partial \mathbb{R}_{+}^{n+1}=\mathbb{R}^{n}$. More precisely, if $u=$ $u(x)$ is a function defined on $\mathbb{R}^{n}$, we consider its harmonic extension $v=v(x, \lambda)$ in $\mathbb{R}_{+}^{n+1}=\mathbb{R}^{n} \times(0,+\infty)$. It is well known (see $\left.[8,12]\right)$ that $u$ is a solution of $(1.1)$ if and only if $v$ satisfies

$$
\begin{cases}\Delta v=0 & \text { in } \mathbb{R}_{+}^{n+1} \\ -\partial_{\lambda} v=f(v) & \text { on } \mathbb{R}^{n}=\partial \mathbb{R}_{+}^{n+1} .\end{cases}
$$

Problem (1.3), associated to the nonlocal equation (1.1), allows to introduce the notions of energy, stability, and global minimality for a solution $u$ of problem (1.1).

Let $\Omega \subset \mathbb{R}_{+}^{n+1}$ be a bounded domain. We denote by

$$
\widetilde{B}_{r}^{+}=\left\{(x, \lambda) \in \mathbb{R}^{2 m+1}: \lambda>0,|(x, \lambda)|<r\right\}
$$

and by $\widetilde{B}_{r}^{+}(x, \lambda)=(x, \lambda)+\widetilde{B}_{r}^{+}$. 
We define the following subset of $\partial \Omega$ :

$$
\partial^{0} \Omega:=\left\{(x, 0) \in \mathbb{R}_{+}^{n+1}: \widetilde{B}_{\varepsilon}^{+}(x, 0) \subset \Omega \text { for some } \varepsilon>0\right\}
$$

and

$$
\partial^{+} \Omega:=\overline{\partial \Omega \cap \mathbb{R}_{+}^{n+1}} .
$$

Given a $C^{1, \alpha}$ nonlinearity $f: \mathbb{R} \rightarrow \mathbb{R}$, for some $0<\alpha<1$, define

$$
G(u)=\int_{u}^{1} f
$$

We have that $G \in C^{2}(\mathbb{R})$ and $G^{\prime}=-f$.

Let $v$ be a $C^{1}(\bar{\Omega})$ function. We consider the energy functional

$$
\mathcal{E}_{\Omega}(v)=\int_{\Omega} \frac{1}{2}|\nabla v|^{2}+\int_{\partial^{0} \Omega} G(v) .
$$

Observe that the potential energy is computed only on the boundary $\partial^{0} \Omega \subset \partial \mathbb{R}_{+}^{n+1}$. This is a quite different situation from the one of interior reactions.

We start by recalling that problem (1.3) can be viewed as the Euler-Lagrange equation associated to the energy functional $\mathcal{E}$.

Definition 1.3. a) We say that a bounded solution $v$ of (1.3) is stable if the second variation of energy $\delta^{2} \mathcal{E} / \delta^{2} \xi$, with respect to perturbations $\xi$ compactly supported in $\overline{\mathbb{R}_{+}^{n+1}}$, is nonnegative. That is, if

$$
Q_{v}(\xi):=\int_{\mathbb{R}_{+}^{n+1}}|\nabla \xi|^{2}-\int_{\partial \mathbb{R}_{+}^{n+1}} f^{\prime}(v) \xi^{2} \geq 0
$$

for every $\xi \in C_{0}^{\infty}\left(\overline{\mathbb{R}_{+}^{n+1}}\right)$.

We say that $v$ is unstable if and only if $v$ is not stable.

b) We say that a bounded solution $u$ of (1.1) in $\mathbb{R}^{2 m}$ is stable (unstable) if its harmonic extension $v$ is a stable (unstable) solution for the problem (1.3).

Another important notion related to the energy functional $\mathcal{E}$ is the one of global minimality.

Definition 1.4. a) We say that a bounded $C^{1}\left(\overline{\mathbb{R}_{+}^{n+1}}\right)$ function $v$ in $\mathbb{R}_{+}^{n+1}$ is a global minimizer of (1.3) if

$$
\mathcal{E}_{\Omega}(v) \leq \mathcal{E}_{\Omega}(v+\xi),
$$

for every bounded domain $\Omega \subset \overline{\mathbb{R}_{+}^{n+1}}$ and every $C^{\infty}$ function $\xi$ with compact support in $\Omega \cup \partial^{0} \Omega$.

b) We say that a bounded $C^{1}$ function $u$ in $\mathbb{R}^{n}$ is a global minimizer of (1.1) if its harmonic extension $v$ is a global minimizer of (1.3). 
Observe that the perturbations $\xi$ do not need to vanish on $\partial^{0} \Omega$, in contrast from interior reactions.

In some references, global minimizers are called "local minimizers", where local stands for the fact that the energy is computed in bounded domains. Clearly, every global minimizer is a stable solution.

In what follows we will assume some or all of the following properties on $f$ :

$$
\begin{gathered}
f \text { is odd; } \\
G \geq 0=G( \pm 1) \text { in } \mathbb{R}, \text { and } G>0 \text { in }(-1,1) ; \\
f^{\prime} \text { is decreasing in }(0,1) .
\end{gathered}
$$

Note that, if (1.8) and (1.9) hold, then $f(0)=f( \pm 1)=0$. Conversely, if $f$ is odd in $\mathbb{R}$, positive with $f^{\prime}$ decreasing in $(0,1)$ and negative in $(1, \infty)$ then $f$ satisfies (1.8), (1.9) and (1.10). Hence, the nonlinearities $f$ that we consider are of "balanced bistable type", while the potentials $G$ are of "double well type". Our three assumptions (1.8), (1.9), (1.10) are satisfied for the scalar Allen-Cahn type equation

$$
(-\Delta)^{1 / 2} u=u-u^{3} \text {. }
$$

In this case we have that $G(u)=(1 / 4)\left(1-u^{2}\right)^{2}$ and (1.8), (1.9), (1.10) hold. The three hypothesis also hold for the Peierls-Nabarro problem

$$
(-\Delta)^{1 / 2} u=\sin (\pi u),
$$

for which $G(u)=(1 / \pi)(1+\cos (\pi u))$.

By a result of Cabré and Solà-Morales [8], assumption (1.9) on $G$ guarantees the existence of an increasing solution, from -1 to 1 , of (1.1) in $\mathbb{R}$. We call these solutions layer solutions. In addition, such an increasing solution is unique up to translations.

The following is the precise result established in [8].

Theorem 1.5 ([8]). Let $f$ be any $C^{1, \alpha}$ function with $0<\alpha<1$ and $G^{\prime}=-f$. Then:

- There exists an increasing solution $u_{0}: \mathbb{R} \rightarrow(-1,1)$ of $(-\Delta)^{1 / 2} u_{0}=f\left(u_{0}\right)$ in $\mathbb{R}$ (that is, a layer solution $u_{0}$ ) if and only if

$$
G^{\prime}(-1)=G^{\prime}(1)=0, \text { and } G>G(-1)=G(1) \text { in }(-1,1) .
$$

- If $f^{\prime}( \pm 1)<0$, then a layer solution of (1.3) is unique up to translations.

- If $f$ is odd and $f^{\prime}( \pm 1)<0$, then every layer solution of (1.3) is odd in $x$ with respect to some half-axis. That is, $u(x+b)=-u(-x+b)$ for some $b \in \mathbb{R}$.

Normalizing the layer solution to vanishing at the origin, we call it $u_{0}$ and its harmonic extension in the half-plane $v_{0}$. Thus we have

$$
\left\{\begin{array}{l}
u_{0}: \mathbb{R} \rightarrow(-1,1) \\
u_{0}(0)=0, \quad u_{0}^{\prime}>0 \\
(-\Delta)^{1 / 2} u_{0}=f\left(u_{0}\right) \quad \text { in } \mathbb{R} .
\end{array}\right.
$$


The monotone bounded solution $u_{0}$ of the Peierls-Nabarro problem (1.12) in $\mathbb{R}$ is explicit. Calling $v_{0}$ its harmonic extension in $\mathbb{R}_{+}^{2}$ we have that

$$
v_{0}(x, \lambda)=\frac{2}{\pi} \arctan \frac{x}{\lambda+1 / \pi} .
$$

In the following theorem, we establish the existence of a saddle-shaped solution for problem (1.1) in every even dimension $n=2 m$. We use the following notations:

$$
\begin{aligned}
& \mathcal{O}:=\left\{x \in \mathbb{R}^{2 m}: s>t\right\} \subset \mathbb{R}^{2 m} \\
& \widetilde{\mathcal{O}}:=\left\{(x, \lambda) \in \mathbb{R}_{+}^{2 m+1}: x \in \mathcal{O}\right\} \subset \mathbb{R}_{+}^{2 m+1} .
\end{aligned}
$$

Note that

$$
\partial \mathcal{O}=\mathcal{C}
$$

We define the cylinder

$$
C_{R, L}=B_{R} \times(0, L),
$$

where $B_{R}$ is the open ball in $\mathbb{R}^{2 m}$ centered at the origin and of radius $R$.

Theorem 1.6. For every dimension $2 m \geq 2$ and every nonlinearity $f$ satisfying (1.8) and (1.9), there exists a saddle solution $u$ of $(-\Delta)^{1 / 2} u=f(u)$ in $\mathbb{R}^{2 m}$, such that $|u|<1$ in $\mathbb{R}^{2 m}$.

Let $v$ be the harmonic extension of the saddle solution $u$ in $\mathbb{R}_{+}^{2 m+1}$. If in addition $f$ satisfies (1.10), then the second variation of the energy $Q_{v}(\xi)$ at $v$, as defined in (1.7), is nonnegative for all function $\xi \in C^{1}\left(\overline{\mathbb{R}_{+}^{2 m+1}}\right)$ with compact support in $\overline{\mathbb{R}_{+}^{2 m+1}}$ and vanishing on $\mathcal{C} \times[0,+\infty)$.

We prove the existence of a saddle solution $u$ for problem (1.1), by proving the existence of a solution $v$ for problem (1.3), with the following properties:

(1) $v$ depends only on the variables $s, t$ and $\lambda$. We write $v=v(s, t, \lambda)$;

(2) $v>0$ for $s>t$;

(3) $v(s, t, \lambda)=-v(t, s, \lambda)$.

Using a variational technique we construct a solution $v$ for the following problem

$$
\begin{cases}\Delta v=0 & \text { in } \widetilde{\mathcal{O}} \\ v>0 & \text { in } \mathcal{\mathcal { O }} \\ v=0 & \text { on } \mathcal{C} \times[0,+\infty) \\ -\partial_{\lambda} v=f(v) & \text { on } \mathcal{O} \times\{\lambda=0\} .\end{cases}
$$

Then, since $f$ is odd, by odd reflection with respect to $\mathcal{C} \times[0,+\infty)$ we obtain a solution $v$ in the whole space which satisfies properties (1), (2), (3). Clearly the function $u(x)=v(x, 0)$ is a saddle solution for problem (1.1). 
To prove this existence result, we will use the following non-sharp energy estimate for $v$. Given $1 / 2 \leq \gamma<1$, there exists $\varepsilon=\varepsilon(\gamma)>0$ such that

$$
\mathcal{E}_{C_{S, S^{\gamma}}}(v) \leq C S^{2 m-\varepsilon} .
$$

In [4, Theorem 1.7], Cabré and the author establish the following sharp energy estimates for saddle-shaped solutions,

$$
\mathcal{E}_{C_{S, S}}(v) \leq C S^{2 m-1} \log S .
$$

Here, (1.14) is not sharp, but it is enough to prove the existence of a saddle solution.

For solutions of problem (1.3) depending only on the coordinates $s, t$ and $\lambda$, problem (1.3) becomes

$$
\begin{cases}-\left(v_{s s}+v_{t t}+v_{\lambda \lambda}\right)-(m-1)\left(\frac{v_{s}}{s}+\frac{v_{t}}{t}\right)=0, & \text { in } \mathbb{R}_{+}^{2 m+1} \\ -\partial_{\lambda} v=f(v) & \text { on } \partial \mathbb{R}_{+}^{2 m+1},\end{cases}
$$

while the energy functional becomes

$$
\begin{aligned}
& \mathcal{E}(v, \Omega) \\
& =c_{m}\left\{\int_{\Omega} s^{m-1} t^{m-1} \frac{1}{2}\left(v_{s}^{2}+v_{t}^{2}+v_{\lambda}^{2}\right) d s d t d \lambda+\int_{\partial^{0} \Omega} s^{m-1} t^{m-1} G(v) d s d t\right\},
\end{aligned}
$$

where $c_{m}$ is a positive constant depending only on $m$-here we have assumed that $\Omega \subset \mathbb{R}^{2 m+1}$ is radially symmetric in the first $m$ variables and also in the last $m$ variables, and we have abused notation by identifying $\Omega$ with its projection in the $(s, t, \lambda)$ variables.

In Section 5, we prove the existence and monotonicity properties of a maximal saddle solution.

To establish these results, we need to introduce a new nonlocal operator $D_{H, \varphi}$, which is the square root of the Laplacian for functions defined in domains $H \subset \mathbb{R}^{n}$ which do not vanish on $\partial H$. We introduce this operator and we establish maximum principles for it, in Section 4.

We define the new variables

$$
\left\{\begin{array}{l}
y=\frac{s+t}{\sqrt{2}} \\
z=\frac{s-t}{\sqrt{2}} .
\end{array}\right.
$$

Note that $|z| \leq y$ and that we may write the Simons cone as $\mathcal{C}=\{z=0\}$.

The following theorem concerns the existence and monotonicity properties of a maximal saddle solution. 
Theorem 1.7. Let $f$ satisfy conditions (1.8), (1.9), and (1.10).

Then, there exists a saddle solution $\bar{u}$ of $(-\Delta)^{1 / 2} \bar{u}=f(\bar{u})$ in $\mathbb{R}^{2 m}$, with $|u|<$ 1 , which is maximal in the following sense. For every solution $u$ of $(-\Delta)^{1 / 2} u=$ $f(u)$ in $\mathbb{R}^{2 m}$, vanishing on the Simons cone and such that $u$ has the same sign as $s-t$, we have

$$
0<u \leq \bar{u} \text { in } \mathcal{O} \text {. }
$$

As a consequence, we also have

$$
0 \leq|u| \leq|\bar{u}| \text { in } \mathbb{R}^{2 m} .
$$

In addition, if $\bar{v}$ is the harmonic extension of $\bar{u}$ in $\mathbb{R}_{+}^{2 m+1}$, then $\bar{v}$ satisfies:

(a) $\partial_{s} \bar{v} \geq 0$ in $\overline{\mathbb{R}_{+}^{2 m+1}}$. Furthermore $\partial_{s} \bar{v}>0$ in $\overline{\mathbb{R}_{+}^{2 m+1}} \backslash\{s=0\}$ and $\partial_{s} \bar{v}=0$ in $\{s=0\}$;

(b) $\partial_{t} \bar{v} \leq 0$ in $\overline{\mathbb{R}_{+}^{2 m+1}}$. Furthermore $\partial_{t} \bar{v}<0$ in $\overline{\mathbb{R}_{+}^{2 m+1}} \backslash\{t=0\}$ and $\partial_{t} \bar{v}=0$ in $\{t=0\}$;

(c) $\partial_{z} \bar{v}>0$ in $\overline{\mathbb{R}_{+}^{2 m+1}} \backslash\{0\}$;

(d) $\partial_{y} \bar{v}>0$ in $\{s>t\} \times[0,+\infty)$.

As a consequence, for every direction $\partial_{\eta}=\alpha \partial_{y}-\beta \partial_{t}$, with $\alpha$ and $\beta$ positive constants, $\partial_{\eta} \bar{v}>0$ in $\{s>t>0\} \times[0,+\infty)$.

Theorem 1.7 above is the analog in [11, Theorem 1.7] for reactions in the interior. In [11] two important ingredients in the proof of the existence and monotonicity properties of the maximal saddle solution are the following. Let $u^{(1)}$ be a saddle solution of $-\Delta u^{(1)}=f\left(u^{(1)}\right)$ in $\mathbb{R}^{2 m}$, with $f$ bistable, and let $u_{0}^{(1)}$ be the layer solution in dimension $n=1$ of $\left(-u_{0}^{(1)}\right)^{\prime \prime}=f\left(u_{0}^{(1)}\right)$ (whose existence is guaranteed by hypothesis (1.9) on $f$ ). Then

i) $u_{0}^{(1)}(|s-t| / \sqrt{2})$ is a supersolution of $-\Delta u^{(1)}=f\left(u^{(1)}\right)$ in $\mathcal{O}$;

ii) $\left|u^{(1)}(x)\right| \leq\left|u_{0}^{(1)}(d(x, \mathcal{C}))\right|=\left|u_{0}^{(1)}\left(\frac{|s-t|}{\sqrt{2}}\right)\right|$ for every $x \in \mathbb{R}^{2 m}$,

where $d(\cdot, \mathcal{C})$ denotes the distance to the Simons cone.

The following proposition establishes the analog for boundary reactions of point i) above.

Proposition 1.8. Let $f$ satisfy hypothesis (1.8), (1.9), (1.10). Let $u_{0}$ be the layer solution, vanishing at the origin, of problem (1.1) in $\mathbb{R}$ and let $v_{0}$ be its harmonic extension in $\mathbb{R}_{+}^{2}$.

Then, the function $v_{0}(z, \lambda)=v_{0}\left(\frac{s-t}{\sqrt{2}}, \lambda\right)$ satisfies

$$
\begin{cases}-\Delta v_{0} \geq 0 & \text { in } \widetilde{\mathcal{O}} \\ -\partial_{\lambda} v_{0} \geq f\left(v_{0}\right) & \text { on } \mathcal{O} \times\{0\}\end{cases}
$$


Concerning point ii) above, estimate (1.18) follows by an important gradient bound of Modica [19] for the classical equation $-\Delta u=f(u)$ in $\mathbb{R}^{n}$.

In the fractional case Cabré and Solà-Morales [8] and Cabré and Sire [6] established a non-local version of the Modica estimate in dimension $n=1$, the analog estimate for dimentsions $n>1$ is still an open problem. Therefore, we are not able to deduce the analog of (1.18) for solutions of the equation $(-\Delta)^{1 / 2} u=f(u)$ in $\mathbb{R}^{2 m}$. For this reason, to give an upper barrier for saddle solutions, that at the same time is a supersolution, we consider the function $\min \left\{K v_{0}(|s-t| / \sqrt{2}, \lambda), 1\right\}$ where $K \geq 1$ is a large constant depending only on $n,\|u\|_{\infty}$, and $f$. Proposition 1.8 implies that this function is a supersolution in $\widetilde{\mathcal{O}}$. Moreover, we will show that there exists $K \geq 1$, depending only on $n,\|u\|_{\infty}$, and $f$, such that if $v$ is a bounded solution of problem (1.3), vanishing on $\mathcal{C} \times[0,+\infty)$, then

$$
|v(x, \lambda)| \leq \min \left\{K v_{0}(|s-t| / \sqrt{2}, \lambda), 1\right\}, \quad \text { for every }(x, \lambda) \in \overline{\mathbb{R}_{+}^{2 m+1}} .
$$

Estimate (1.19) follows by regularity results established in [8].

In section 6 , we prove the following theorem concerning the asymptotic behaviour at infinity for a class of solutions which contains saddle-shaped solutions.

Theorem 1.9. Let $f$ satisfy conditions (1.8), (1.9), and (1.10), and let $u$ be a bounded solution of $(-\Delta)^{1 / 2} u=f(u)$ in $\mathbb{R}^{2 m}$ such that $u \equiv 0$ on $\mathcal{C}, u>0$ in $\mathcal{O}=\{s>t\}$ and $u$ is odd with respect to $\mathcal{C}$.

Then, denoting $U(x):=u_{0}((s-t) / \sqrt{2})=u_{0}(z)$ we have,

$$
u(x)-U(x) \rightarrow 0 \quad \text { and } \quad \nabla u(x)-\nabla U(x) \rightarrow 0,
$$

uniformly as $|x| \rightarrow \infty$. That is,

$$
\|u-U\|_{L^{\infty}\left(\mathbb{R}^{2 m} \backslash B_{R}\right)}+\|\nabla u-\nabla U\|_{L^{\infty}\left(\mathbb{R}^{2 m} \backslash B_{R}\right)} \rightarrow 0 \text { as } R \rightarrow \infty .
$$

Our proof of Theorem 1.9 follows the one given by Cabre and Terra in [11], and uses a compactness argument based on translations of the solutions, combined with two crucial Liouville-type results for nonlinear equations in the half-space and in a quarter of space.

Finally, in Section 7 we establish that saddle-shaped solutions are unstable in dimension $2 m=4$ and $2 m=6$.

Theorem 1.10. Let $f$ satisfy conditions (1.8), (1.9), (1.10). Then, every bounded solution $u$ of $(-\Delta)^{1 / 2} u=f(u)$ in $\mathbb{R}^{2 m}$ such that $u=0$ on the Simons cone $\mathcal{C}=\{s=t\}$ and $u$ has the same sign as $s-t$, is unstable in dimension $2 m=4$ and $2 m=6$.

Instability in dimension $2 m=2$ follows by a result of Cabré and Solà Morales [8] which asserts that every stable solution of (1.1) in dimension $n=2$ is onedimensional. This is the analog of the conjecture of De Giorgi in dimension $n=2$ for the half-Laplacian. 
In [10], Cabré and Terra proved instability in dimension $2 m=4$ for saddleshaped solutions of the classical equation $-\Delta u=f(u)$ in $\mathbb{R}^{4}$. A crucial ingredient in the proof of this result is the pointwise estimate (1.18).

However, in dimension $2 m=6$, this estimate is not enough to prove instability and thus Cabré and Terra used a more precise argument, based on some monotonicity properties and asymptotic behaviour of a maximal saddle solution.

Since, as said before, we cannot prove the analog of (1.18) for solutions of the equation $(-\Delta)^{1 / 2} u=f(u)$, here we follow the argument introduced by Cabré and Terra in dimension $2 m=6$, both for the case $2 m=4$ and $2 m=6$.

Using this approach, the crucial ingredients in the proof of Theorem 1.10 are:

i) the equation satisfied by $\bar{v}_{z}$, where $\bar{v}$ is the harmonic extension of the maximal saddle solution $\bar{u}$ in $\mathbb{R}_{+}^{2 m+1}$

ii) a monotonicity property of $\bar{v}$;

iii) the asymptotic behaviour at infinity of $\bar{v}$.

The paper is organized as follows:

- In Section 2 we prove Theorem 1.6 concerning the existence of a saddle solution for the equation (1.1) in every dimension $2 m$.

- In Section 3, we give a supersolution and a subsolution for the square root of the Laplacian in a domain $H \subset \mathbb{R}^{n}$. In particular we prove Proposition 1.8.

- In Section 4, we introduce the operator $D_{H, \varphi}$ and we establish maximum principles for it.

- In Section 5, we prove the existence of a maximal saddle solution $\bar{u}$ and its monotonicity properties (Theorem 1.7).

- In Section 6, we prove Theorem 1.9, concerning the asymptotic behaviour of saddle solutions.

- In Section 7, we prove Theorem 1.10 about the instability of saddle solutions in dimensions $2 m=4$ and $2 m=6$.

ACKNOWLEDGEMENTS. The author wishes to thank Xavier Cabre for his guidance and for fruitful discussions on the topic of this paper.

\section{Existence of a saddle solution in $\mathbb{R}^{2 m}$}

In this section we prove the existence of a saddle solution $u$ for problem (1.1), by proving the existence of a solution $v$ for problem (1.3) with the following properties:

(1) $v$ depends only on the variables $s, t$ and $\lambda$. We write $v=v(s, t, \lambda)$;

(2) $v>0$ for $s>t$;

(3) $v(s, t, \lambda)=-v(t, s, \lambda)$.

We recall that we have defined the sets:

$$
\mathcal{O}=\left\{x \in \mathbb{R}^{2 m}: s>t\right\} \subset \mathbb{R}^{2 m}, \quad \widetilde{\mathcal{O}}=\left\{(x, \lambda) \in \mathbb{R}_{+}^{2 m+1}: x \in \mathcal{O}\right\} \subset \mathbb{R}_{+}^{2 m+1} .
$$


Let $B_{R}$ be the open ball in $\mathbb{R}^{2 m}$ centered at the origin and of radius $R$. We will consider the open bounded sets

$$
\begin{gathered}
\mathcal{O}_{R}:=\mathcal{O} \cap B_{R}=\left\{s>t,|x|^{2}=s^{2}+t^{2}<R^{2}\right\} \subset \mathbb{R}^{2 m} . \\
\widetilde{\mathcal{O}}_{R, L}:=\left(\mathcal{O} \cap B_{R}\right) \times(0, L)=\left\{(x, \lambda) \in \mathbb{R}_{+}^{2 m+1}: s>t,|x|^{2}=s^{2}+t^{2}<R^{2}, \lambda<L\right\} .
\end{gathered}
$$

Note that

$$
\partial \mathcal{O}_{R}=\left(\mathcal{C} \cap \bar{B}_{R}\right) \cup\left(\partial B_{R} \cap \mathcal{O}\right) .
$$

Before giving the proof of Theorem 1.6, we recall some results established in [8] concerning the regularity of weak solutions of problem (1.3). Cabré and SolàMorales (see [8, Lemma 2.2]) proved that every bounded weak solution $v$ of problem (1.3) with $f \in C^{1, \alpha}$, satisfies $v \in C^{2, \alpha}$, for all $0<\alpha<1$. This result was deduced using the auxiliary function

$$
w(x, \lambda)=\int_{0}^{\lambda} v(x, t) d t
$$

which is a solution of the Dirichlet problem

$$
\left\{\begin{array}{ll}
-\Delta w=f(v(x, 0)) & \text { in } \mathbb{R}_{+}^{2 m+1} \\
w(x, 0)=0 & \text { on } \partial \mathbb{R}_{+}^{2 m+1}
\end{array} .\right.
$$

Applying standard regularity results for the Dirichlet problem above, they deduce regularity for the solution $v$ of problem (1.3). Moreover, using standard elliptic estimates for bounded harmonic functions, we have that the following gradient bound for $v$ holds:

$$
|\nabla v(x, \lambda)| \leq \frac{C}{1+\lambda} \quad \text { for every }(x, \lambda) \in \overline{\mathbb{R}_{+}^{2 m+1}} .
$$

We define now the sets

$$
\widetilde{L}^{2}\left(\widetilde{\mathcal{O}}_{R, L}\right)=\left\{v \in L^{2}\left(\widetilde{\mathcal{O}}_{R, L}\right): v=v(s, t, \lambda) \text { a.e. }\right\}
$$

and

$$
\widetilde{H}_{0}^{1}\left(\widetilde{\mathcal{O}}_{R, L}\right)=\left\{v \in H^{1}\left(\widetilde{\mathcal{O}}_{R, L}\right): v \equiv 0 \text { on } \partial^{+} \widetilde{\mathcal{O}}_{R, L}, v=v(s, t, \lambda) \text { a.e. }\right\} .
$$

They are, respectively, the set of $L^{2}$ functions in the bounded open set $\widetilde{\mathcal{O}}_{R, L}$ which depend only on $s, t$, and $\lambda$, and the set of $H^{1}$ functions in the bounded open set $\widetilde{\mathcal{O}}_{R, L}$ which depend only on $s, t$ and $\lambda$ and which vanish on the positive boundary $\partial^{+} \widetilde{\mathcal{O}}_{R, L}$ in the weak sense.

We recall that the inclusion $\widetilde{H}_{0}^{1}\left(\widetilde{\mathcal{O}}_{R, L}\right) \subset \subset \widetilde{L}^{2}\left(\partial^{0} \widetilde{\mathcal{O}}_{R, L}\right)$ is compact (see [8]). Indeed, let $v \in \widetilde{H}_{0}^{1}\left(\widetilde{\mathcal{O}}_{R, L}\right)$. Since $v \equiv 0$ on $\partial^{+} \widetilde{\mathcal{O}}_{R, L}$, we can extend $v$ to be 
identically 0 in $\mathbb{R}_{+}^{2 m+1} \backslash \widetilde{\mathcal{O}}_{R, L}$, so that $v \in \widetilde{H}^{1}\left(\mathbb{R}_{+}^{2 m+1}\right)=\left\{v \in H^{1}\left(\mathbb{R}_{+}^{2 m+1}\right): v=\right.$ $v(s, t, \lambda)$ a.e. $\}$. We have

$$
\begin{aligned}
\int_{\partial^{0} \widetilde{\mathcal{O}}_{R, L}}|v(x, 0)|^{2} d x & =-\int_{\mathbb{R}_{+}^{n+1}} \partial_{\lambda}\left(|v|^{2}\right) \\
& =-2 \int_{\mathbb{R}_{+}^{n+1}} v \partial_{\lambda} v \leq\left. C\|v\|\right|_{\widetilde{L}^{2}\left(\widetilde{\mathcal{O}}_{R, L}\right)}\|v\|_{\widetilde{H}^{1}\left(\widetilde{\mathcal{O}}_{R, L}\right)} .
\end{aligned}
$$

Now, the compactness of the inclusion, follows from the fact that since $v \equiv 0$ on $\partial^{+} \widetilde{\mathcal{O}}_{R, L}$ a.e., then $\widetilde{H}_{0}^{1}\left(\widetilde{\mathcal{O}}_{R, L}\right) \subset \subset \widetilde{L}^{2}\left(\partial^{0} \widetilde{\mathcal{O}}_{R, L}\right)$ is compact (to see this it is enough to extend $v$ to be identically zero in a $A \backslash \widetilde{\mathcal{O}}_{R, L}$, where $A \subset \mathbb{R}_{+}^{n+1}$ is a Lipschitz set containing $\widetilde{\mathcal{O}}_{R, L}$ ).

We can now give the proof of Theorem 1.6.

Proof of Theorem 1.6. As already mentioned, we prove the existence of a solution $v$ for the problem (1.3) such that $v=v(s, t, \lambda)$ and $v(s, t, \lambda)=-v(-t, s, \lambda)$. The space $\widetilde{H}_{0}^{1}\left(\widetilde{\mathcal{O}}_{R, L}\right)$, defined above, is a weakly closed subspace of $H^{1}\left(\widetilde{\mathcal{O}}_{R, L}\right)$.

Consider the energy functional in $\widetilde{\mathcal{O}}_{R, L}$,

$$
\mathcal{E}_{\widetilde{\mathcal{O}}_{R, L}}(v)=\int_{\widetilde{\mathcal{O}}_{R, L}} \frac{1}{2}|\nabla v|^{2}+\int_{\partial^{0} \widetilde{\mathcal{O}}_{R, L}} G(v) \quad \text { for every } v \in \widetilde{H}_{0}^{1}\left(\widetilde{\mathcal{O}}_{R, L}\right) .
$$

Next, we prove the existence of a minimizer of this functional among functions in $\widetilde{H}_{0}^{1}\left(\widetilde{\mathcal{O}}_{R, L}\right)$. Recall that we assume condition (1.9) on $G$, that is,

$$
G( \pm 1)=0 \text { and } G>0 \text { in }(-1,1) .
$$

We define a continuous function $\widetilde{G}$ which coincides with $G$ in $[-1,1]$ and satisfies the following properties:

- $\widetilde{G}=G$ in $[-1,1]$,

- $\widetilde{G}>0$ in $\mathbb{R} \backslash[-1,1]$,

- $\widetilde{G}$ is even,

- $\widetilde{G}$ has linear growth at infinity.

We consider the new energy functional

$$
\widetilde{\mathcal{E}}_{\widetilde{\mathcal{O}}_{R, L}}(v)=\int_{\widetilde{\mathcal{O}}_{R, L}} \frac{1}{2}|\nabla v|^{2}+\int_{\partial^{0} \widetilde{\mathcal{O}}_{R, L}} \widetilde{G}(v) \quad \text { for every } \quad v \in \widetilde{H}_{0}^{1}\left(\widetilde{\mathcal{O}}_{R, L}\right) .
$$

Note that every minimizer $w$ of $\widetilde{\mathcal{E}}_{\widetilde{\mathcal{O}}_{R, L}}(\cdot)$ in $\widetilde{H}_{0}^{1}\left(\widetilde{\mathcal{O}}_{R, L}\right)$ such that $-1 \leq w \leq 1$ is also a minimizer of $\mathcal{E}_{\widetilde{\mathcal{O}}_{R, L}}(\cdot)$ in the set

$$
\left\{v \in \widetilde{H}_{0}^{1}\left(\widetilde{\mathcal{O}}_{R, L}\right):-1 \leq v \leq 1\right\} .
$$


We show that $\widetilde{\mathcal{E}}_{\widetilde{\mathcal{O}}_{R, L}}(\cdot)$ admits a minimizer in $\widetilde{H}_{0}^{1}\left(\widetilde{\mathcal{O}}_{R, L}\right)$. Indeed, by the properties of $\widetilde{G}$, it follows that $\widetilde{\mathcal{E}}_{\widetilde{\mathcal{O}}_{R, L}}(\cdot)$ is well-defined, bounded below and coercive in $\widetilde{H}_{0}^{1}\left(\widetilde{\mathcal{O}}_{R, L}\right)$. Hence, using the compactness of the inclusion $\widetilde{H}_{0}^{1}\left(\widetilde{\mathcal{O}}_{R, L}\right) \subset \subset$ $\widetilde{L}^{2}\left(\partial^{0} \widetilde{\mathcal{O}}_{R, L}\right)$, taking a minimizing sequence $\left\{v_{R, L}^{k}\right\} \in \widetilde{H}_{0}^{1}\left(\widetilde{\mathcal{O}}_{R, L}\right)$ and a subsequence convergent in $\widetilde{L}^{2}\left(\partial^{0} \widetilde{\mathcal{O}}_{R, L}\right)$, we conclude that $\widetilde{\mathcal{E}}_{\widetilde{\mathcal{O}}_{R, L}}(\cdot)$ admits an absolute minimizer $v_{R, L}$ in $\widetilde{H}_{0}^{1}\left(\widetilde{\mathcal{O}}_{R, L}\right)$.

Note moreover that, without loss of generality, we may assume that $0 \leq$ $v_{R, L}^{k} \leq 1$ in $\widetilde{\mathcal{O}}_{R, L}$ because, if not, we can replace the minimizing sequence $v_{R, L}^{k}$ with the sequence $\min \left\{\left|v_{R, L}^{k}\right|, 1\right\} \in \widetilde{H}_{0}^{1}\left(\widetilde{\mathcal{O}}_{R, L}\right)$. Indeed, it is also minimizing because $\widetilde{G}$ is even and $\widetilde{G} \geq \widetilde{G}(1)$. Then an absolute minimizer $v_{R, L}$ is such that $0 \leq v_{R, L} \leq 1$ in $\widetilde{\mathcal{O}}_{R, L}$

Next, we can consider perturbations $v_{R, L}+\xi$ of $v_{R, L}$, with $\xi$ depending only on $s, t$ and $\lambda$, and having compact support in $\widetilde{\mathcal{O}}_{R, L} \cap\{t>0\}$. In particular $\xi$ vanishes in a neighborhood of $\{t=0\}$. Since the problem $(1.3)$ in $(s, t, \lambda)$ coordinates is the first variation of $\mathcal{E}_{\widetilde{\mathcal{O}}_{R, L}}(v)$-recall that $\mathcal{E}$ has the form (1.16) on $\widetilde{H}_{0}^{1}$ functionsand the equation is not singular away from $\{s=0\}$ and $\{t=0\}$, we deduce that $v_{R, L}$ is a solution of (1.15) in $\widetilde{\mathcal{O}}_{R, L} \cap\{t>0\}$.

We now prove that $v_{R, L}$ is also a solution in all of $\widetilde{\mathcal{O}}_{R, L}$, that is, also across $\{t=0\}$. To see this for dimensions $2 m+1 \geq 5$, let $\xi_{\varepsilon}$ be a smooth function of $t$ alone being identically 0 in $\{t<\varepsilon / 2\}$ and identically 1 in $\{t>\varepsilon\}$. Thus we have that $\left|\nabla \xi_{\varepsilon}\right| \leq 2 / \varepsilon$. Let $\varphi \in C_{0}^{\infty}\left(\widetilde{\mathcal{O}}_{R, L} \cup \partial^{0} \widetilde{\mathcal{O}}_{R, L}\right)$, we multiply the equation $-\Delta v_{R, L}=0$ by $\varphi \xi_{\varepsilon}$ and integrate by parts to obtain

$$
\int_{\widetilde{\mathcal{O}}_{R, L}} \nabla v_{R, L} \nabla \varphi \xi_{\varepsilon}+\int_{\widetilde{\mathcal{O}}_{R, L} \cap\{t<\varepsilon\}} \nabla v_{R, L} \varphi \nabla \xi_{\varepsilon}+\int_{\partial^{0} \widetilde{\mathcal{O}}_{R, L}} \partial_{\lambda} v_{R, L} \varphi \xi_{\varepsilon}=0 .
$$

Reminding that $v_{R, L}$ satisfies the Neumann condition $-\partial_{\lambda} v_{R, L}=f\left(v_{R, L}\right)$ on $\partial^{0} \widetilde{\mathcal{O}}_{R, L}$, we get

$$
\int_{\widetilde{\mathcal{O}}_{R, L}} \nabla v_{R, L} \nabla \varphi \xi_{\varepsilon}+\int_{\widetilde{\mathcal{O}}_{R, L} \cap\{t<\varepsilon\}} \nabla v_{R, L} \varphi \nabla \xi_{\varepsilon}=\int_{\partial^{0} \widetilde{\mathcal{O}}_{R, L}} f\left(v_{R, L}\right) \varphi \xi_{\varepsilon} .
$$

We conclude by seeing that the second integral on the left-hand side goes to zero as $\varepsilon \rightarrow 0$. Indeed, by Cauchy-Schwartz inequality,

$$
\begin{aligned}
& \left|\int_{\widetilde{\mathcal{O}}_{R, L} \cap\{t<\varepsilon\}} \nabla v_{R, L} \varphi \nabla \xi_{\varepsilon} d x d \lambda\right|^{2} \\
& \leq C \int_{\widetilde{\mathcal{O}}_{R, L} \cap\{t<\varepsilon\}}\left|\nabla v_{R, L}\right|^{2} d x d \lambda \int_{\widetilde{\mathcal{O}}_{R, L} \cap\{t<\varepsilon\}}\left|\nabla \xi_{\varepsilon}\right|^{2} d x d \lambda .
\end{aligned}
$$


Since $\left|\nabla \xi_{\varepsilon}\right|^{2} \leq C / \varepsilon^{2},\left|\widetilde{\mathcal{O}}_{R, L} \cap\{t<\varepsilon\}\right| \leq C_{R} \varepsilon^{m} L$, and $m \geq 2$, the second factor in the previous bound, is bounded independently of $\varepsilon$. At the same time, the first factor tends to zero as $\varepsilon \rightarrow 0$, since $\left|\nabla v_{R, L}\right|^{2}$ is integrable in $\widetilde{\mathcal{O}}_{R, L}$.

In dimension $2 m+1=3$, the previous proof does not apply and we argue as follows. We consider perturbations $\xi \in \tilde{H}_{0}^{1}\left(\mathcal{\mathcal { O }}_{R, L}\right)$ which do not vanish on $\{t=0\}$. Considering the first variation of energy and integrating by parts, we find that the boundary flux $s^{m-1} t^{m-1} \partial_{t} v_{R, L}=\partial_{t} v_{R, L}$ (here $m-1=0$ ) must be identically 0 on $\{t=0\}$. This implies that $v_{R, L}$ is a solution also across $\{t=0\}$.

We have established the existence of a solution $v_{R, L}$ in $\widetilde{\mathcal{O}}_{R, L}$ with $0 \leq v_{R, L} \leq$ 1. Considering the odd reflection of $v_{R, L}$ with respect to $\mathcal{C} \times \mathbb{R}^{+}$,

$$
v_{R, L}(s, t, \lambda)=-v_{R, L}(t, s, \lambda),
$$

we obtain a solution in $B_{R} \backslash\{0\} \times(0, L)$. Using the same cut-off argument as above, but choosing now $1-\xi_{\varepsilon}$ to have support in the ball of radius $\varepsilon$ around 0 , we conclude that $v_{R, L}$ is also solution around 0 , and hence in all of $B_{R} \times(0, L)$. Here, the cut-off argument also applies in dimension 3.

We now wish to pass to the limit in $R$ and $L$, and obtain a solution in all of $\mathbb{R}_{+}^{2 m+1}$. Let $S>0, L^{\prime}>0$ and consider the family $\left\{v_{R, L}\right\}$ of solutions in $B_{S+2} \times\left[0, L^{\prime}+2\right]$, with $R>S+2$ and $L>L^{\prime}+2$. Since $\left|v_{R, L}\right| \leq 1$, regularity results proved in Lemma 2.2 of [8] , applied in $B_{2} \times[0,2]$ where $B_{2}$ is centered at points in $\bar{B}_{S} \times\left[0, L^{\prime}\right]$, give a uniform $C^{2, \alpha}\left(\bar{B}_{S} \times\left[0, L^{\prime}\right]\right.$ ) bound for $v_{R, L}$ (uniform with respect to $R$ and $L$ ). We have

$$
\left|\nabla v_{R, L}\right| \leq C \quad \text { in } B_{S} \times\left[0, L^{\prime}\right], \quad \text { for all } R>S+2, L>L^{\prime}+2
$$

for some constant $C$ independent of $S, R, L$ and $L^{\prime}$. Moreover since $v_{R, L}$ is harmonic and bounded we have that

$$
\left|\nabla v_{R, L}(x, \lambda)\right| \leq \frac{C}{\lambda} \quad \text { in } B_{R} \times(1, L) .
$$

Choose now $L=R^{\gamma}$, with $1 / 2<\gamma<1$ (this choice will be used later to prove that the solution that we construct is not identically zero). By the Arzelà-Ascoli Theorem, a subsequence of $\left\{v_{R, R^{\gamma}}\right\}$ converges in $C^{2}\left(\bar{B}_{S} \times\left[0, S^{\gamma}\right]\right)$ to a solution in $B_{S} \times\left(0, S^{\gamma}\right)$. Taking $S=1,2,3, \ldots$ and making a Cantor diagonal argument, we obtain a sequence $v_{R_{j}, R_{j}^{\gamma}}$ converging in $C_{l o c}^{2}\left(\mathbb{R}_{+}^{2 m+1}\right)$ to a solution $v \in C^{2}\left(\mathbb{R}_{+}^{2 m+1}\right)$. By construction we have found a solution $v$ in $\mathbb{R}_{+}^{2 m+1}$ depending only on $s, t$ and $\lambda$, such that $v(s, t, \lambda)=-v(t, s, \lambda),|v| \leq 1$ and $v \geq 0$ in $\{s>t\}$. We want to prove now that $|v|<1$. Indeed, remind that $v$ satisfies

$$
\begin{cases}\Delta v=0 & \text { in } \mathbb{R}_{+}^{2 m+1} \\ -\partial_{\lambda} v=f(v) & \text { on } \partial \mathbb{R}^{2 m+1} .\end{cases}
$$

Since $f(1)=0$ and $v$ is not identically 1 (because $v \equiv 0$ on $\mathcal{C} \times \mathbb{R}^{+}$), using that $v \leq 1$ and applying the maximum principle and Hopf's Lemma, we conclude that $v<1$. In the same way we prove that $v>-1$. 
It only remains to prove that $v \not \equiv 0$ in $\mathbb{R}_{+}^{2 m+1}$. Then, the strong maximum principle and Hopf's Lemma lead to $v>0$ in $\{s>t\} \times \mathbb{R}^{+}$since $f(0)=0$ and $v \geq 0$ in $\{s>t\} \times \mathbb{R}^{+}$.

To prove that $v \not \equiv 0$ in $\mathbb{R}_{+}^{2 m+1}$, we establish an energy estimate for the saddle solution constructed above, which is not sharp, but it is enough to prove $v \not \equiv 0$ in $\mathcal{O}=\{s>t\} \times \mathbb{R}^{+}$.

We use a comparison argument, based on the minimality property of $v_{R, L}$ in the set $\widetilde{\mathcal{O}}_{R, L}$.

Let $1 / 2<\gamma<1$ as above and $\beta$ be a positive real number depending only on $\gamma$ and such that $1 / 2 \leq \beta<\gamma<1$. Let $S<R-2$, then $S^{\gamma}<L$ since we have chosen $L=R^{\gamma}$. We consider a $C^{1}$ function $g: \widetilde{\mathcal{O}}_{S, S^{\gamma}} \rightarrow \mathbb{R}$ defined as follows:

$$
g(x, \lambda)=g(s, t, \lambda)=\eta(s, t) \min \left\{1, \frac{s-t}{\sqrt{2}}\right\}+(1-\eta(s, t)) v_{R, L}(s, t, \lambda),
$$

where $\eta$ is a smooth function depending only on $r^{2}=s^{2}+t^{2}$ such that $\eta \equiv 1$ in $B_{S-1}$ and $\eta \equiv 0$ outside $B_{S}$ and thus $|\nabla \eta| \leq 2$. Observe that $g$ agrees with $v_{R, L}$ on the lateral boundary of $\widetilde{\mathcal{O}}_{S, S^{\gamma}}$ and $g$ is identically 1 inside $\left(\mathcal{O}_{S-1} \cap\{(s-t) / \sqrt{2}>\right.$ $1\}) \times\left(0, S^{\gamma}\right)$.

Next we consider a $C^{1}$ function $\xi:\left(0, S^{\gamma}\right) \rightarrow(0,+\infty)$, such that

$$
\xi(\lambda)= \begin{cases}1 & \text { if } 0<\lambda \leq S^{\gamma}-S^{\beta} \\ \frac{\log S^{\gamma}-\log \lambda}{\log S^{\gamma}-\log \left(S^{\gamma}-S^{\beta}\right)} & \text { if } S^{\gamma}-S^{\beta}<\lambda \leq S^{\gamma} .\end{cases}
$$

Then, we define $w: \widetilde{\mathcal{O}}_{S, S^{\gamma}} \rightarrow(-1,1)$ as follows

$$
w(x, \lambda)=\xi(\lambda) g(x, \lambda)+[1-\xi(\lambda)] v_{R, L}(x, \lambda) .
$$

For simplicity of notations we set $\hat{\mathcal{O}}:=\left(\mathcal{O}_{S-1} \cap\{(s-t) / \sqrt{2}>1\}\right) \times\left(0, S^{\gamma}-S^{\beta}\right)$. Observe that $w$ agree with $v_{R, L}$ on $\partial^{+} \widetilde{\mathcal{O}}_{S, S^{\gamma}}$ and $w \equiv 1$ in $\hat{\mathcal{O}}$. We extend $w$ to be identically equal to $v_{R, L}$ in $\widetilde{\mathcal{O}}_{R, L} \backslash \widetilde{\mathcal{O}}_{S, S^{\gamma}}$. By minimality of $v_{R, L}$ in $\widetilde{\mathcal{O}}_{R, L}$, we have

$$
\mathcal{E}_{\widetilde{\mathcal{O}}_{R, L}}\left(v_{R, L}\right) \leq \mathcal{E}_{\widetilde{\mathcal{O}}_{R, L}}(w) .
$$

Thus, since $w=v_{R, L}$ in $\widetilde{\mathcal{O}}_{R, L} \backslash \widetilde{\mathcal{O}}_{S, S^{\gamma}}$, we get

$$
\mathcal{E}_{\widetilde{\mathcal{O}}_{S, S \gamma}}\left(v_{R, L}\right) \leq \mathcal{E}_{\widetilde{\mathcal{O}}_{S, S \gamma}}(w) .
$$

We give now an estimate for $\mathcal{E}_{\widetilde{\mathcal{O}}_{S, S \gamma}}(w)$. First, observe that, since $w \equiv 1$ on $\mathcal{O}_{S-1} \cap$ $\{(s-t) / \sqrt{2}>1\}$, then

$$
\begin{aligned}
\int_{\mathcal{O}_{S}} G(w) & =\int_{\mathcal{O}_{S} \backslash\left(\mathcal{O}_{S-1} \cap\{(s-t) / \sqrt{2}>1\}\right)} G(w) \\
& \leq C\left|\mathcal{O}_{S} \backslash\left(\mathcal{O}_{S-1} \cap\{(s-t) / \sqrt{2}>1\}\right)\right| \leq C S^{2 m-1}
\end{aligned}
$$


Next, we give a bound for the Dirichlet energy of $w$. We have

$$
\begin{aligned}
\int_{\widetilde{\mathcal{O}}_{S, S \gamma}}|\nabla w(x, \lambda)|^{2} d x d \lambda= & \int_{\widetilde{\mathcal{O}}_{S, S^{\gamma}-S^{\beta}}}|\nabla w(x, \lambda)|^{2} d x d \lambda \\
& +\int_{\widetilde{\mathcal{O}}_{S, S \gamma} \backslash \widetilde{\mathcal{O}}_{S, S \gamma-S^{\beta}}}|\nabla w(x, \lambda)|^{2} d x d \lambda .
\end{aligned}
$$

Since $w \equiv 1$ in $\hat{\mathcal{O}}$, and $\left|\widetilde{\mathcal{O}}_{S, S^{\gamma}-S^{\beta}} \backslash \hat{\mathcal{O}}\right| \leq C S^{2 m-1+\gamma}$, we get

$$
\int_{\widetilde{\mathcal{O}}_{S, S^{\gamma}}}|\nabla w(x, \lambda)|^{2} d x d \lambda \leq C S^{2 m-1+\gamma}+\int_{\widetilde{\mathcal{O}}_{S, S^{\gamma} \backslash \widetilde{\mathcal{O}}_{S, S \gamma}-S^{\beta}}}|\nabla w(x, \lambda)|^{2} d x d \lambda .
$$

Consider now the integral on the right-hand side of (2.9). By the definition (2.6) of $w$, we have that

$|\nabla w(x, \lambda)|^{2} \leq\left|\xi^{\prime}(\lambda)\right|^{2}\left[g(x, \lambda)+v_{R, L}(x, \lambda)\right]^{2}+\left\{|\nabla g|^{2}+\left|\nabla v_{R, L}(x, \lambda)\right|^{2}\right\}[1+\xi(\lambda)]^{2}$

Integrating in $\widetilde{\mathcal{O}}_{S, S \gamma} \backslash \widetilde{\mathcal{O}}_{S, S \gamma-S^{\beta}}$, using that $g,|\nabla g|, v$, and $\xi$ are bounded, the definition of $\xi$, the gradient bound (2.5) for $v_{R, L}$, and the fact that $\nabla g$ vanishes in $\left(\mathcal{O}_{S-1} \cap\{(s-t) / \sqrt{2}>1\}\right) \times\left(0, S^{\gamma}\right)$, we get

$$
\begin{aligned}
& \int_{\widetilde{\mathcal{O}}_{S, S} \gamma \backslash \tilde{\mathcal{O}}_{S, S^{\gamma}-S^{\beta}}}|\nabla w(x, \lambda)|^{2} \\
& \leq C \int_{\mathcal{O}_{S}} \int_{S^{\gamma}-S^{\beta}}^{S^{\gamma}}\left|\xi^{\prime}(\lambda)\right|^{2} d \lambda d x+C S^{2 m-1+\gamma} \\
& \quad+C \int_{\mathcal{O}_{S}} \int_{S^{\gamma}-S^{\beta}}^{S^{\gamma}} \frac{1}{\lambda^{2}} d \lambda d x+C S^{2 m-1+\gamma} \\
& \leq C\left[\frac{1}{\left(\log \frac{S^{\gamma}}{S^{\gamma}-S^{\beta}}\right)^{2}}+1\right] \int_{\mathcal{O}_{S}} \int_{S^{\gamma}-S^{\beta}}^{S^{\gamma}} \frac{1}{\lambda^{2}} d \lambda d x+C S^{2 m-1+\gamma} \\
& \leq C S^{2 m}\left[\frac{1}{\left(-\log \left(1-S^{\beta-\gamma}\right)\right)^{2}}+1\right]\left[\frac{1}{S^{\gamma}-S^{\beta}}-\frac{1}{S^{\gamma}}\right]+C S^{2 m-1+\gamma} \\
& \leq C S^{2 m} \cdot S^{2(\gamma-\beta)} \cdot S^{-\gamma}+C S^{2 m-1+\gamma} \leq C S^{2 m+\gamma-2 \beta}+C S^{2 m-1+\gamma},
\end{aligned}
$$

where $C$ denotes different positive constants independent on $S$.

Combining (2.7), (2.9) and (2.10), we get

$$
\mathcal{E}_{\widetilde{\mathcal{O}}_{S, S \gamma}}(w) \leq C\left(S^{2 m-1}+S^{2 m-1+\gamma}+S^{2 m+\gamma-2 \beta}\right) .
$$

Since, by hypothesis, $\gamma$ and $\beta=\beta(\gamma)$ satisfy $1 / 2 \leq \beta<\gamma<1$, then there exists $\varepsilon=\varepsilon(\gamma)>0$ such that

$$
\mathcal{E}_{\widetilde{\mathcal{O}}_{S, S^{\gamma}}}(w) \leq C S^{2 m-\varepsilon} .
$$


Thus by minimality of $v_{R, L}$, we get

$$
\mathcal{E}_{\widetilde{\mathcal{O}}_{S, S \gamma}}\left(v_{R, L}\right) \leq C S^{2 m-\varepsilon} .
$$

We now let $R$ and $L=R^{\gamma}$ tend to infinity to obtain

$$
\mathcal{E}_{\widetilde{\mathcal{O}}_{S, S^{\gamma}}}(v) \leq C S^{2 m-\varepsilon} .
$$

Note that this bound, after odd reflection with respect to $\mathcal{C}$, leads to the energy bound (1.14)

$$
\mathcal{E}_{C_{S, S^{\gamma}}}(v) \leq C S^{2 m-\varepsilon}
$$

Using this estimate we prove the claim. Suppose that $v \equiv 0$. Then we would have

$$
c_{m} G(0) S^{2 m}=\mathcal{E}_{C_{S, S \gamma}}(v) \leq C S^{2 m-\varepsilon} .
$$

This is a contradiction for $S$ large, and thus $v \not \equiv 0$.

We give now the proof of the last part of the statement, that is, we prove stability of saddle-shaped solutions under perturbations vanishing on $\mathcal{C} \times(0,+\infty)$.

Since $f(0)=0$, concavity leads to $f^{\prime}(w) \leq f(w) / w$ for all real numbers $w \in(0,1)$. Hence we have

$$
\begin{cases}-\Delta v=0 & \text { in } \widetilde{\mathcal{O}} \\ -\partial_{\lambda} v \geq f^{\prime}(v) v & \text { on } \mathcal{O} \times\{0\} .\end{cases}
$$

By a simple argument (see the proof of [1, Proposition 4.2]), it follows that the value of the quadratic form $Q_{v}(\xi)$ is nonnegative for all $\xi \in C^{1}$ with compact support in $\widetilde{\mathcal{O}} \cup \partial^{0} \widetilde{\mathcal{O}}$ (and not necessarily depending only on $s, t$ and $\lambda$ ). Indeed, multiply the equation $-\Delta v=0$ by $\xi^{2} / v$, where $\xi \in C^{1}\left(\mathbb{R}_{+}^{2 m+1}\right)$ with compact support in $\widetilde{\mathcal{O}} \cup \partial^{0} \widetilde{\mathcal{O}}$, and integrate by parts in $\widetilde{\mathcal{O}}$, we get:

$$
\begin{aligned}
0= & \int_{\widetilde{\mathcal{O}}}(-\Delta v) \frac{\xi^{2}}{v}=\int_{\widetilde{\mathcal{O}}} \nabla v \cdot \nabla \xi \frac{2 \xi}{v} \\
& -\int_{\widetilde{\mathcal{O}}}|\nabla v|^{2} \frac{\xi^{2}}{v^{2}}+\int_{\partial^{0} \mathcal{O}} \frac{\xi^{2}}{v} \frac{\partial v}{\partial \lambda} \\
\leq & \int_{\widetilde{\mathcal{O}}}|\nabla \xi|^{2}-\int_{\partial^{0} \mathcal{O}} f^{\prime}(v) \xi^{2}=Q_{v}(\xi) .
\end{aligned}
$$

By an approximation argument, the same holds for all $\xi \in C^{1}$ with compact support in the closure of $\widetilde{\mathcal{O}}$ and vanishing on $\mathcal{C} \times \mathbb{R}^{+}$. Finally, by odd symmetry with respect to $\mathcal{C} \times \mathbb{R}^{+}$, the same is true for all $C^{1}$ functions $\xi$ with compact support in $\overline{\mathbb{R}_{+}^{2 m+1}}$ and vanishing on $\mathcal{C} \times \mathbb{R}^{+}$. 
Remark 2.1. Observe that, if $\gamma \rightarrow 1$, estimate (2.11) tends to

$$
\mathcal{E}_{C_{S, S}}(v) \leq C S^{2 m} .
$$

This is a not sharp energy estimate, indeed in [4, Theorem 1.7], Cabré and the author prove that saddle solutions $v$ satisfy

$$
\mathcal{E}_{C_{S, S}}(v) \leq C S^{2 m-1} \log S .
$$

\section{Supersolution and subsolution for $A_{1 / 2}$}

In [9], Cabre and Tan introduced the operator $A_{1 / 2}$, which is the square root of the Laplacian for functions defined on a bounded set and that vanish on the boundary. Let $u$ be defined in a bounded set $H \subset \mathbb{R}^{n}$ and $u \equiv 0$ on $\partial H$. Consider the harmonic extension $v$ of $u$ in the half-cylinder $H \times(0, \infty)$ vanishing on the lateral boundary $\partial H \times[0, \infty)$. Define the operator $A_{1 / 2}$ as follows

$$
A_{1 / 2} u:=-\partial_{\lambda} v_{\mid H \times\{0\}} .
$$

Then, since $\partial_{\lambda} v$ is harmonic and also vanishes on the lateral boundary, as for the case of the all space, the Dirichlet-Neumann map of the harmonic extension $v$ on the bottom of the half cylinder is the square root of the Laplacian. That is, we have the property:

$$
A_{1 / 2} \circ A_{1 / 2}=-\Delta_{H}
$$

where $-\Delta_{H}$ is the Laplacian in $H$ with zero Dirichlet boundary value on $\partial H$.

Hence, we can study the problem

$$
\begin{cases}A_{1 / 2} u=f(u) & \text { in } H \\ u=0 & \text { on } \partial H \\ u>0 & \text { in } H,\end{cases}
$$

by studying the local problem

$$
\begin{cases}-\Delta v=0 & \text { in } \quad \Omega=H \times(0, \infty) \\ v=0 & \text { on } \quad \partial_{L} \Omega=\partial H \times[0, \infty) \\ v>0 & \text { in } \quad \Omega \\ -\partial_{\lambda} v=f(v) & \text { on } \quad H \times\{0\} .\end{cases}
$$

In [9] some results (Lemma 3.2.3 and Lemma 3.2.4) need to assume that $H$ is bounded. But for our aim, definition (3.1) is enough and it can be given also in the case that $H$ is not bounded. Thus, we can consider problem (3.2) and (3.3) for a general open set $H \subset \mathbb{R}^{n}$. 
In this section we give a subsolution and supersolution for the problem

$$
\begin{cases}A_{1 / 2} u=f(u) & \text { in } \mathcal{O} \\ u=0 & \text { on } \partial \mathcal{O} \\ u>0 & \text { in } \mathcal{O} .\end{cases}
$$

In what follows it will be useful to use the new variables:

$$
\left\{\begin{array}{l}
y=\frac{s+t}{\sqrt{2}} \\
z=\frac{s-t}{\sqrt{2}}
\end{array}\right.
$$

Note that $|z| \leq y$ and that we may write the Simons cone as $\mathcal{C}=\{z=0\}$.

If we take into account these new variables, problem (1.15) becomes

$$
\begin{cases}v_{y y}+v_{z z}+v_{\lambda \lambda}+\frac{2(m-1)}{y^{2}-z^{2}}\left(y v_{y}-z v_{z}\right)=0 & \text { in } \mathbb{R}_{+}^{2 m+1} \\ -\partial_{\lambda} v=f(v) & \text { on } \partial \mathbb{R}_{+}^{2 m+1} .\end{cases}
$$

We give the definition of supersolution and subsolution for problem (3.2) by using the associated local formulation (3.3).

Definition 3.1. a) We say that a function $w$, defined on $H \times[0,+\infty), w \equiv 0$ on $\partial H \times[0,+\infty)$ is a supersolution (subsolution) for problem (3.3) if

$$
\begin{cases}-\Delta w \geq(\leq) 0 & \text { in } H \times(0,+\infty) \\ -\partial_{\lambda} w \geq(\leq) f(w) & \text { on } H \times\{0\}\end{cases}
$$

b) We say that a function $u$, defined on $H, u \equiv 0$ on $\partial H$, is a supersolution (subsolution) for problem (3.2) if its harmonic extension $v$ such that $v \equiv 0$ on $\partial H \times[0,+\infty)$, is a supersolution (subsolution) for problem (3.3).

Lemma 3.2. The following assertions are equivalent:

i) $u$ is a subsolution (supersolution) for problem (3.2);

ii) there exists an extension $w$ of $u$ on $H \times(0,+\infty)$ vanishing on $\partial H \times(0,+\infty)$, such that $w$ is a subsolution (supersolution) for problem (3.3).

Proof. The first implication i) $\Rightarrow$ ii) is trivial.

It remains to show that ii) $\Rightarrow$ i). We consider the case of supersolution (the argument for subsolution is analog). Suppose that there exists a function $w$ defined on $\mathbb{R}_{+}^{n+1}$ such that:

$$
\begin{cases}-\Delta w \geq 0 & \text { in } H \times(0,+\infty) \\ w \equiv 0 & \text { on } \partial H \times(0,+\infty) \\ w>0 & \text { in } H \times(0,+\infty) \\ w(x, 0)=u(x) & \text { on } H \times\{0\} \\ -\partial_{\lambda} w \geq f(w) & \text { on } H \times\{0\} .\end{cases}
$$


Now consider the harmonic extension $v$ of $u$ in $H \times(0,+\infty)$, with $v \equiv 0$ on $\partial H \times$ $(0,+\infty)$. Then by the maximum principle we have that $v \leq w$ in $H \times(0,+\infty)$ This implies that

$$
-\partial_{\lambda} v \geq-\partial_{\lambda} w \text { on } \partial H \times(0,+\infty)
$$

and hence that

$$
-\partial_{\lambda} v \geq f(v) \text { on } \partial H \times(0,+\infty) .
$$

We recall that in [8] it is proven that, under hypothesis (1.9), there exists a layer solution (i.e., a monotone increasing solution, from -1 to 1 ), for problem (1.3) in dimension $n=1$. Normalizing it to vanish at $\{x=0\}$, we call it $u_{0}$ (see (1.13)).

Moreover we remind that $|s-t| / \sqrt{2}$ is the distance to the Simons cone (see [10]).

We can give now the following proposition. The first part of the statement, which gives a supersolution for problem (3.2) in $H=\mathcal{O}$, is equivalent to Proposition 1.8 in the Introduction.

Proposition 3.3. Let $f$ satisfy hypothesis (1.8), (1.9), (1.10). Let $u_{0}$ be the layer solution, vanishing at the origin, of problem $(1.1)$ in $\mathbb{R}$.

Then, the function $u_{0}(z)=u_{0}(s-t) / \sqrt{2}$ is a supersolution of problem (3.2) in the set $H=\mathcal{O}=\{s>t\}$.

Remark 3.4. We observe that, if $f$ satisfies hypothesis (1.8), (1.9), (1.10), then $f(\rho) / \rho$ is non-increasing in $(0,1)$. Indeed, given $0<\rho<1$, there exists $\rho_{1}$, with $0<\rho_{1}<\rho$, such that

$$
\frac{f(\rho)}{\rho}=\frac{f(\rho)-f(0)}{\rho-0}=f^{\prime}\left(\rho_{1}\right)>f^{\prime}(\rho) .
$$

Therefore

$$
\left(\frac{f(\rho)}{\rho}\right)^{\prime}=\frac{f^{\prime}(\rho) \rho-f(\rho)}{\rho^{2}}=\frac{f^{\prime}(\rho)-f^{\prime}\left(\rho_{1}\right)}{\rho}<0
$$

Proof of Proposition 3.3. We begin by considering the function $v_{0}((s-t) / \sqrt{2}, \lambda)$ and we show that it is a supersolution of the problem (3.3) in the set $\widetilde{\mathcal{O}}$.

First, we remind that the problem $(3.3)$ in the $(s, t, \lambda)$ variables reads

$$
\begin{cases}-\left(v_{s s}+v_{t t}+v_{\lambda \lambda}\right)-(m-1)\left(\frac{v_{s}}{s}+\frac{v_{t}}{t}\right)=0 & \text { in } \widetilde{\mathcal{O}} \\ v=0 & \text { on } \mathcal{C} \times[0,+\infty) \\ -\partial_{\lambda} v=f(v) & \text { on } \widetilde{\mathcal{O}} \cap\{\lambda=0\} \\ v>0 & \text { in } \widetilde{\mathcal{O}} .\end{cases}
$$

By a direct computation, we have that $v_{0}((s-t) / \sqrt{2}, \lambda)$ is superharmonic in the set $\{(s, t, \lambda): s>t>0\}$ and satisfies the Neumann condition $-\partial_{\lambda} v=f(v)$. In 
dimension $2 m+1 \geq 5$ there is nothing else to be checked, by a cut-off argument used as in (2.2).

In dimension $2 m+1=3, v_{0}((s-t) / \sqrt{2}, \lambda)$ is a supersolution in $\widetilde{\mathcal{O}}$ because the outer flux $-\partial_{t} v_{0}((s-t) / \sqrt{2}, \lambda)=\partial_{x} v_{0}((s-t) / \sqrt{2}, \lambda)>0$ is positive.

Remark 3.5. Observe that in dimension $2 m+1=3, v_{0}((s-t) / \sqrt{2}, \lambda)$ is a solution of problem (1.3) away from the sets $\{s=0\},\{t=0\}$, while in higher dimensions it is a strict supersolution.

Corollary 3.6. Let $f$ satisfy hypothesis (1.8), (1.9), (1.10). Let $u_{0}$ be the layer solution, vanishing at the origin, of problem (1.1) in $\mathbb{R}$ and suppose $K \geq 1$.

Then, the function $\min \left\{K u_{0}(z), 1\right\}=\min \left\{K u_{0}(s-t / \sqrt{2}), 1\right\}$ is a supersolution of problem (3.2) in the set $\mathcal{O}=\{s>t\}$.

Proof. Proceeding as in the proof of Proposition 3.3, we consider the function $\min \left\{K v_{0}(z, \lambda), 1\right\}$. To prove that it is a supersolution of problem (3.3) in $\widetilde{\mathcal{O}}$, it is enough to prove that it is a supersolution of problem (3.3) in the set $\{(x, \lambda) \in \widetilde{\mathcal{O}}$ : $\left.K v_{0}(z, \lambda)<1\right\}$.

First of all, in the proof of Proposition 3.3, we have seen that $v_{0}(z, \lambda)$ is superharmonic in $\widetilde{\mathcal{O}}$, and thus $\min \left\{K v_{0}(z, \lambda), 1\right\}=K v_{0}(z, \lambda)$ is superharmonic in the set $\left\{(x, \lambda) \in \widetilde{\mathcal{O}}: K v_{0}(z, \lambda)<1\right\}$.

Moreover

$$
-\partial_{\lambda}\left(K v_{0}(z, 0)\right)=K f\left(v_{0}(z, 0)\right) \quad \text { on }\left\{(x, 0) \in \widetilde{\mathcal{O}}: K v_{0}(z, 0)<1\right\} .
$$

By Remark 3.4, we have that $f(u) / u$ is decreasing and then for every $K \geq 1$ we get

$$
\frac{K f\left(u_{0}\right)}{K u_{0}}=\frac{f\left(u_{0}\right)}{u_{0}} \geq \frac{f\left(K u_{0}\right)}{K u_{0}} \quad \text { if } K u_{0}<1 .
$$

This let us to conclude the proof, indeed

$$
\begin{aligned}
& -\partial_{\lambda}\left(K v_{0}(z, 0)\right)=K f\left(v_{0}(z, 0)\right) \geq f\left(K v_{0}(z, 0)\right) \\
& \text { on }\left\{(x, 0) \in \widetilde{\mathcal{O}}: K v_{0}(z, 0)<1\right\} .
\end{aligned}
$$

\section{The operator $D_{H, \varphi}$ and maximum principles}

In what follows we need to introduce a new nonlocal operator $D_{H, \varphi}$, which is the analogue of $A_{1 / 2}$ but it can be applied to functions which do not vanish on the boundary of $H$.

Suppose that $u$ and $\varphi$ are functions defined in $\bar{H} \subset \mathbb{R}^{n}$, such that $u=\varphi$ on $\partial H$. As in the case of $A_{1 / 2}$ we want to consider the harmonic extension $v$ of $u$ in the cylinder $\Omega=H \times(0,+\infty)$ and we have to give Dirichlet data on the lateral 
boundary of the cylinder $\partial_{L} \Omega=\partial H \times(0,+\infty)$. We do it in the following way: we put $v(x, \lambda)=\varphi(x)$ for every $(x, \lambda) \in \partial_{L} \Omega$.

As before we define $D_{H, \varphi}$ as follows:

$$
D_{H, \varphi} u:=-\partial_{\lambda} v_{\mid \Omega \times\{0\}} .
$$

We observe that, since $v$ is independent on $\lambda$ on $\partial_{L} \Omega$, we have $v_{\lambda}=0$ on the lateral boundary. Thus, we can apply the operator $A_{1 / 2}$ to $v_{\lambda}(x, 0)$ and we get, as before

$$
A_{1 / 2} \circ D_{H, \varphi}=-\Delta_{H, \varphi}
$$

where $-\Delta_{H, \varphi}$ is the Laplacian in $H$ with Dirichlet boundary value $\varphi$.

If we have a nonlocal problem of the type

$$
\begin{cases}D_{H, \varphi} u=f(u) & \text { in } H \\ u=\varphi & \text { on } \partial H,\end{cases}
$$

then it can be restated in the local problem

$$
\begin{cases}-\Delta v=0 & \text { in } \Omega \\ v(x, \lambda)=\varphi(x) & \text { on } \partial_{L} \Omega \\ -\partial_{\lambda} v=f(v) & \text { on } H \times\{0\}\end{cases}
$$

Observe that the operator $D_{H, \varphi}$ coincides with $A_{1 / 2}$ if the boundary data $\varphi$ is identically zero.

Next, we give some maximum principles for the operator $D_{H, \varphi}$.

Lemma 4.1. Let $\Omega=H \times \mathbb{R}^{+}$be a cylinder in $\mathbb{R}_{+}^{n+1}$, where $H \subset \mathbb{R}^{n}$ is a bounded domain. Let $\left.v \in C^{2}(\Omega) \cap C^{(} \bar{\Omega}\right)$ be a bounded harmonic function in $\Omega$. Then,

$$
\inf _{\Omega} v=\inf _{\partial \Omega} v .
$$

Proof. Substracting a constant from $v$, we may assume that $v$ is nonnegative on $\partial \Omega$ and we need to show $v \geq 0$ in $\Omega$.

We follow a classical argument based on the construction of a strictly positive harmonic function $\psi$ in $\Omega$ tending to infinity as $|(x, \lambda)| \rightarrow \infty$. We proceed in the following way.

First, since $H \subset \mathbb{R}^{n}$ is bounded, there exists a ball $B_{R}$ of radius $R$ in $\mathbb{R}^{n}$ such that $\bar{H} \subset B_{R}$. Let $\mu_{R}$ and $\phi_{R}$ be, respectively, the first eigenvalue and the corresponding eigenfunction of the Laplacian $-\Delta$ in $B_{R}$ with 0 -Dirichlet value on $\partial B_{R}$.

We define the function $\psi: B_{R} \times \mathbb{R}^{+} \rightarrow \mathbb{R}$ as follows

$$
\psi(x, \lambda)=\phi_{R}(x) e^{\sqrt{\mu_{R} \lambda}} .
$$


Then the restriction of $\psi$ in $\Omega$ is a strictly positive harmonic function.

Moreover, since $\phi_{R}$ is strictly positive, we have that

$$
\lim _{|(x, \lambda)| \rightarrow+\infty} \psi(x, \lambda)=\lim _{\lambda \rightarrow+\infty} \psi(x, \lambda)=+\infty .
$$

We consider now the function $w=v / \psi$. Then $w$ satisfies

$$
\begin{cases}-\Delta w-2 \frac{\nabla \psi}{\psi} \cdot \nabla w=0 & \text { in } \Omega \\ w \geq 0 & \text { on } \partial \Omega .\end{cases}
$$

Note that $w$ has the same sign as $v$. In addition, by (4.2), $w(x, \lambda) \rightarrow 0$ as $|(x, \lambda)| \rightarrow$ $+\infty$ and thus, by the strong maximum principle (applied, by a contradiction argument, to a possible negative minimum) $w \geq 0$ in $\Omega$, which implies $v \geq 0$ in $\Omega$.

From the previous result we deduce the following lemma.

Lemma 4.2. Assume that $u \in C^{2}(H) \cap C(\bar{H})$ satisfies

$$
\begin{cases}D_{H, \varphi} u+c(x) u \geq 0 & \text { in } H, \\ u=\varphi & \text { on } \partial H,\end{cases}
$$

where $H$ is a bounded domain in $\mathbb{R}^{n}$ and $c(x) \geq 0$ in $H$. Suppose that $\varphi \geq 0$ on $\partial H$. Then $u \geq 0$ in $H$.

Proof. Consider the harmonic extension $v$ of $u$ in $\Omega=H \times(0,+\infty)$ with Dirichlet data $v(x, \lambda)=\varphi(x)$ on the lateral boundary $\partial_{L} \Omega=\partial H \times(0,+\infty)$ (as in the definition of the operator $D_{H, \varphi}$ ). We prove that $v \geq 0$ in $\Omega$, then in particular $u \geq 0$ in $H$.

Suppose by contradiction that $v$ is negative somewhere in $\Omega \times \mathbb{R}^{+}$. Since $v$ is harmonic, by Lemma 4.1 the $\inf _{\Omega} v<0$ will be achieved at some point $\left(x_{0}, 0\right) \in$ $H \times\{0\}$. Thus, we have

$$
\inf _{\Omega} v=v\left(x_{0}, 0\right)<0 .
$$

By Hopf's lemma,

$$
v_{\lambda}\left(x_{0}, 0\right)>0 .
$$

It follows

$$
-v_{\lambda}\left(x_{0}, 0\right)=D_{H, \varphi} v\left(x_{0}, 0\right)<0 .
$$

Therefore, since $c \geq 0$,

$$
D_{H, \varphi} v\left(x_{0}, 0\right)+c\left(x_{0}\right) v\left(x_{0}, 0\right)<0 .
$$

This is a contradiction with the hypothesis $D_{H, \varphi} u+c(x) u \geq 0$.

The following corollary follows directly by the previous lemma. 
Corollary 4.3. Let $H$ be a bounded domain in $\mathbb{R}^{n}$. Suppose that $u_{1}$ and $u_{2}$ are two bounded functions, $u_{1}, u_{2} \in C^{2}(H) \cap C(\bar{H})$, which satisfy

$$
\begin{cases}D_{H, \varphi} u_{1} \leq D_{H, \varphi} u_{2} & \text { in } H \\ u_{1}=u_{2}=\varphi & \text { on } \partial H .\end{cases}
$$

Then, $u_{1} \leq u_{2}$ in $H$.

We conclude this section with the following strong maximum principle.

Lemma 4.4. Assume that $u \in C^{2}(H) \cap C(\bar{H})$ satisfies

$$
\begin{cases}D_{H, \varphi} u+c(x) u \geq 0 & \text { in } H, \\ u \geq 0 & \text { in } H, \\ u=\varphi & \text { on } \partial H,\end{cases}
$$

where $\Omega$ is a smooth bounded domain in $\mathbb{R}^{n}$ and $c \in L^{\infty}(H)$. Suppose $\varphi \geq 0$ on $\partial H$.

Then, either $u>0$ in $H$, or $u \equiv 0$ in $H$.

Proof. The proof is similar to the one of Lemma 4.2.

Consider the harmonic extension $v$ of $u$ in $\Omega=H \times[0,+\infty)$ with lateral boundary data $v=\varphi$ on $\partial_{L} \Omega$. We observe that $v \geq 0$ in $\Omega$. Suppose that $v \not \equiv 0$ but $v=0$ somewhere in $\Omega$. Then there exists a minimum point $x_{0} \in H$ such that $v\left(x_{0}, 0\right)=0$. Hence by Hopf's lemma we see that $\partial_{\lambda} v\left(x_{0}, 0\right)>0$. This implies that $D_{H, \varphi} u\left(x_{0}\right)+c\left(x_{0}\right) u\left(x_{0}\right)<0$, since $v\left(x_{0}, 0\right)=u\left(x_{0}\right)=0$, which is a contradiction.

\section{Maximal saddle solution and monotonicity properties}

In this section we prove Theorem 1.7 concerning the existence and monotonicity properties of a maximal saddle solution. In the proof we will use that every saddle solution $u$ of $(-\Delta)^{1 / 2} u=f(u)$ is bounded above by the function $u_{b}(z)=\min \{1, K|u(z)|\}$ where $z=|s-t| / \sqrt{2}$ is the distance to the Simons cone and $K$ is a large constant. Let $R>0$ and consider the open region

$$
T_{R}=\left\{x \in \mathbb{R}^{2 m}: t<s<R\right\} .
$$

Note that $T_{R} \supset \mathcal{O}_{R}=\mathcal{O} \cap B_{R}$.

Let, as before, $v$ be the harmonic extension of a saddle solution $u$ in the halfspace $\mathbb{R}_{+}^{2 m+1}$. The regularity results given in [8] give a uniform upper bound for $|\nabla v|($ see $(2.1))$. Then, since $v=0$ on $\mathcal{C} \times \mathbb{R}^{+}=\{z=0\} \times \mathbb{R}^{+}$, there exists a constant $C$, depending only on $n,\|u\|_{\infty}$, and $\|f\|_{C^{1}}$, such that

$$
|v(x, \lambda)|=|v(y, z, \lambda)| \leq C|z|, \quad \text { for every }(x, \lambda) \in \overline{\mathbb{R}_{+}^{2 m+1}} .
$$

In particular, we have that $|u(x)|=|v(x, 0)| \leq C|z|$ for every $x \in \mathbb{R}^{2 m}$. 
Observe that there exists a real number $K \geq 1$ such that

$$
\min \{1, C|z|\} \leq \min \left\{1, K\left|u_{0}(z)\right|\right\} \quad \text { for every } z .
$$

Indeed it is enough to choose

$$
K \geq \max \left\{C / u_{0}^{\prime}(0), 1 / u_{0}\left(C^{-1}\right)\right\} .
$$

This is possible since the quantities $u_{0}^{\prime}(0)$ and $u_{0}\left(C^{-1}\right)$ are strictly positive.

If we choose $K$ as in (5.2), then the harmonic extension $v$ in $\mathbb{R}_{+}^{2 m+1}$ of every saddle solution $u$ of (1.1) satisfies

$$
|v(x, \lambda)| \leq \min \left\{1, K\left|u_{0}(z)\right|\right\} \quad \text { for every }(x, \lambda) \in \overline{\mathbb{R}_{+}^{2 m+1}} .
$$

We define

$$
u_{b}(z):=\min \left\{1, K\left|u_{0}(z)\right|\right\}
$$

where $K$ satisfies (5.2). Note that $u_{b}=0$ on $\mathcal{C} \cap \overline{T_{R}}$.

Lemma 5.1. Let $f$ satisfies conditions (1.8), (1.9), (1.10).

Then, there exists a positive solution $\bar{u}_{R}$ of

$$
\begin{cases}D_{T_{R}, u_{b}} u=f(u) & \text { in } T_{R} \\ u=u_{b} & \text { on } \partial T_{R} .\end{cases}
$$

which is maximal in $T_{R}$ in the following sense. We have that $\bar{u}_{R} \geq u$ in $T_{R}$ (and hence in $\mathcal{O}_{R}$ ) for every bounded solution $u$ of $(-\Delta)^{1 / 2} u=f(u)$ in $\mathbb{R}^{2 m}$ that vanishes on the Simons cone and has the same sign as $s-t$. In addition $\bar{u}_{R}$ depends only on $s$ and $t$.

Proof. We construct a sequence of solutions of linear problems involving the operator $D_{T_{R}, u_{b}}$ and, by the iterative use of the maximum principle, we prove that this sequence is non increasing and it converges to the maximal solution $\bar{u}_{R}$.

We set

$$
L w:=\left(D_{T_{R}, u_{b}}+a\right) w, \text { and } g(w):=f(w)+a w,
$$

where $a$ is a positive constant chosen such that $g^{\prime}(w)=f^{\prime}(w)+a$ is positive for every $w$.

Next we define a sequence of functions $\bar{u}_{R, j}$ as follows. We set

$$
\bar{u}_{R, 0}(x):=u_{b}=\min \left\{1, K u_{0}(z)\right\}, \quad \text { for every } x \in T_{R},
$$

and we define $\bar{u}_{R, j+1}$ to be the solution of the linear problem

$$
\left\{\begin{aligned}
L \bar{u}_{R, j+1} & =g\left(\bar{u}_{R, j}\right) & & \text { in } T_{R} \\
\bar{u}_{R, j+1} & =u_{b} & & \text { on } \partial T_{R} .
\end{aligned}\right.
$$


Since $L$ is obtained by adding a positive constant to $D_{T_{R}, u_{b}}$, it satisfies the maximum principles (Lemma 4.2 and Corollary 4.3) and hence the above problem admits a unique solution $\bar{u}_{R, j+1}=\bar{u}_{R, j+1}(x)$. Furthermore (and here we argue by induction), since the problem and its data are invariant by orthogonal transformations in the first (respectively, in the last) $m$ variables $x_{i}$, the solution $\bar{u}_{R, j+1}$ depends only on $s$ and $t$.

First, observe that by Corollary 3.6, the function $\bar{u}_{R, 0}=\min \left\{1, K u_{0}(z)\right\}$ is a supersolution of problem $L w=g(w)$, i.e., $L \bar{u}_{R_{0}} \geq g\left(\bar{u}_{R, 0}\right)$. This implies that $L \bar{u}_{R, 1}=g\left(\bar{u}_{R, 0}\right) \leq L \bar{u}_{R, 0}$ and then $\bar{u}_{R, 1} \leq \bar{u}_{R, 0} \leq 1$ in $T_{R}$. Moreover $u_{b} \geq 0$ on $\partial T_{R}$ and therefore, by Lemma $4.2, \bar{u}_{R, 1} \geq 0$ in $T_{R}$.

Assume now that $0 \leq \bar{u}_{R, j} \leq \bar{u}_{R, j-1} \leq 1$ for some $j \geq 1$. By the choice of $a$, we have $g\left(\bar{u}_{R, j}\right) \leq g\left(\bar{u}_{R, j-1}\right)$. We get

$$
L \bar{u}_{R, j+1}=g\left(\bar{u}_{R, j}\right) \leq g\left(\bar{u}_{R, j-1}\right)=L \bar{u}_{R, j} .
$$

Again by the maximum principle (Corollary (4.3)) $\bar{u}_{R, j+1} \leq \bar{u}_{R, j}$. Besides, $\bar{u}_{R, j+1} \geq 0$ since $g\left(\bar{u}_{R, j}\right) \geq 0$. Therefore, by induction we have proven that the sequence $\bar{u}_{R, j}$ is nonincreasing, that is

$$
1 \geq \bar{u}_{R, 0}(x) \geq \bar{u}_{R, 1}(x) \geq \cdots \geq \bar{u}_{R, j}(x) \geq \bar{u}_{R, j+1}(x) \geq \cdots \geq 0 .
$$

By monotone convergence, this sequence converges to a nonnegative solution in $T_{R}, \bar{u}_{R}$, which depends only on $s$ and $t$, and such that $\bar{u}_{R}=u_{b}(z)$ on $\partial T_{R}$. Thus, the strong maximum principle (Lemma 4.4) leads to $\bar{u}_{R}>0$ in $T_{R}$.

Moreover, $\bar{u}_{R}$ is maximal with respect to any bounded solution $u,|u|<1$ in $\mathbb{R}^{2 m}$, that vanishes on the Simons cone and has the same sign as $s-t$. Indeed, let $\bar{v}_{R, 1}$ be the harmonic extension of $\bar{u}_{R, 1}$ in $T_{R} \times \mathbb{R}^{+}$which is equal to $u_{b}$ on the lateral boundary $\partial T_{R} \times \mathbb{R}^{+}$. It is the solution of the following problem

$$
\begin{cases}\Delta \bar{v}_{R, 1}=0 & \text { in } T_{R} \times \mathbb{R}^{+} \\ \bar{v}_{R, 1}=u_{b} & \text { on } \partial T_{R} \times \mathbb{R}^{+} \\ -\partial_{\lambda} \bar{v}_{R, 1}+a \bar{v}_{R, 1}=g\left(\bar{u}_{R, 0}\right)=g\left(u_{b}\right) & \text { on } T_{R} \times\{0\} .\end{cases}
$$

Consider now $v$ the harmonic extension of $u$ in $\mathbb{R}_{+}^{2 m+1}$. Then the restriction of $v$ to $T_{R}$, which we still call $v$, is the solution of the problem

$$
\begin{cases}\Delta v=0 & \text { in } T_{R} \times \mathbb{R}^{+} \\ -\partial_{\lambda} v+a v=g(u) & \text { on } T_{R} \times\{0\} .\end{cases}
$$

Recall that by (5.3), we have that $v \leq u_{b}$ in $\overline{\mathbb{R}_{+}^{2 m+1}}$. Since $g$ is increasing, then the difference $v-\bar{v}_{R, 1}$ is a solution of

$$
\begin{cases}\Delta\left(v-\bar{v}_{R, 1}\right)=0 & \text { in } T_{R} \times \mathbb{R}^{+} \\ v-\bar{v}_{R, 1}=v-u_{b} \leq 0 & \text { on } \partial T_{R} \times \mathbb{R}^{+} \\ -\partial_{\lambda}\left(v-\bar{v}_{R, 1}\right)+a\left(v-\bar{v}_{R, 1}\right)=g(u)-g\left(u_{b}\right) \leq 0 & \text { on } T_{R} \times\{0\}\end{cases}
$$


We claim that $v \leq \bar{v}_{R, 1}$ in $T_{R} \times[0,+\infty)$. Indeed, suppose by contradiction that $v-\bar{v}_{R, 1}$ is positive somewhere in $T_{R} \times[0,+\infty)$. Then, by the maximum principle (Lemma 4.2), $\sup \left(v-\bar{v}_{R, 1}\right)>0$ will be achieved at some point $\left(x_{0}, 0\right) \in T_{R} \times\{0\}$. By Hopf's Lemma and since $a$ is positive, we would have

$$
-\partial_{\lambda}\left(v-\bar{v}_{R, 1}\right)\left(x_{0}, 0\right)+a\left(v-\bar{v}_{R, 1}\right)\left(x_{0}, 0\right)>0 .
$$

This is a contradiction with the last inequality of (5.8). Thus we have proved that $v \leq \bar{v}_{R, 1}$ in $T_{R} \times \mathbb{R}^{+}$.

Suppose now that $v \leq \bar{v}_{R, j}$. Arguing as before, we consider the problem satisfied by $\left(v-\bar{v}_{R, j+1}\right)$. Using the maximum principle and Hopf's Lemma we deduce that $v \leq \bar{v}_{R, j+1}$ in $T_{R} \times[0,+\infty)$. By induction, $v \leq \bar{v}_{R, j}$ for every $j$ and, in particular, $u \leq \bar{u}_{R, j}$ for every $j$. Then,

$$
u \leq \bar{u}_{R}=\lim _{j \rightarrow \infty} \bar{u}_{R, j} \quad \text { in } T_{R}
$$

The following are monotonicity results for the maximal solution constructed above.

Lemma 5.2. Let $\bar{u}_{R}$ be the function constructed in Lemma 5.1. Let $\bar{v}_{R}$ be the harmonic function in $T_{R} \times(0,+\infty)$ such that $\bar{v}_{R}(x, 0)=\bar{u}_{R}(x)$ for every $x \in T_{R}$ and $v(x, \lambda)=u_{b}(x)$ for every $(x, \lambda) \in \partial T_{R} \times(0,+\infty)$.

Then $\partial_{t} \bar{v}_{R} \leq 0$.

Proof. We consider the nonincreasing sequence of function $\bar{u}_{R, j}$ constructed in the proof of Lemma 5.1. We set $\bar{v}_{R, 0}(x, \lambda)=\bar{u}_{R, 0}(x)=\min \left\{1, K u_{0}(z)\right\}$ for every $(x, \lambda) \in \mathbb{R}_{+}^{2 m+1}$ and, for every $j \geq 1$ we call $\bar{v}_{R, j}$ the harmonic extension of $\bar{u}_{R, j}$ in $T_{R} \times(0,+\infty)$ such that $\bar{v}_{R, j}(x, \lambda)=u_{b}(x)$ for every $(x, \lambda) \in \partial T_{R} \times(0,+\infty)$.

The function $\bar{v}_{R, j}$ is a solution in coordinates $s$ and $t$ of the problem

$$
\begin{cases}\partial_{s s} \bar{v}_{R, j}+\partial_{t t} \bar{v}_{R, j}+\partial_{\lambda \lambda} \bar{v}_{R, j}+\frac{(m-1)}{s} \partial_{s} \bar{v}_{R, j}+\frac{(m-1)}{t} \partial_{t} \bar{v}_{R, j}=0 \\ & \text { in } T_{R} \times(0, \infty) \\ \bar{v}_{R, j}=u_{b} & \text { on } \partial T_{R} \times(0,+\infty), \\ -\partial_{\lambda} \bar{v}_{R, j}+a \bar{v}_{R, j}=g\left(\bar{v}_{R, j-1}\right) & \text { on } T_{R} \times\{0\} .\end{cases}
$$

Differentiating with respect to $t$ we get:

$$
\begin{cases}-\Delta\left(\partial_{t} \bar{v}_{R, j}\right)+\frac{(m-1)}{t^{2}} \partial_{t} \bar{v}_{R, j}=0 & \text { in } T_{R} \times(0, \infty) \\ -\partial_{\lambda}\left(\partial_{t} \bar{v}_{R, j}\right)+a \partial_{t} \bar{v}_{R, j}=g^{\prime}\left(\bar{v}_{R, j-1}\right) \partial_{t} \bar{v}_{R, j-1} & \text { on } T_{R} \times\{0\} .\end{cases}
$$

We observe that $\partial_{t} \bar{v}_{R, j} \leq 0$ on $\partial T_{R} \times(0,+\infty)$. Indeed $\bar{v}_{R, j} \equiv 0$ on $\left(\mathcal{C} \cap \partial T_{R}\right) \times$ $(0,+\infty)$ and $\bar{v}_{R, j}>0$ inside $T_{R} \times(0,+\infty)$. Then, $\partial_{t} \bar{v}_{R, j} \leq 0$ on $\{t=s<$ $R\} \times(0,+\infty)$.

Moreover $\bar{v}_{R, j}=\min \left\{K u_{0}(z), 1\right\}=\min \left\{K u_{0}((R-t) / \sqrt{2}), 1\right\}$ on $\{t<s=$ $R\}$ and thus $\partial_{t} \bar{v}_{R, j}=-K / \sqrt{2} \dot{u}_{0}((R-t) / \sqrt{2}) \leq 0$ on $\{t<s=R\} \times(0,+\infty)$. 
Now, we argue by induction. First, recall that

$$
\bar{v}_{R, 0}=\min \left\{K u_{0}(z), 1\right\}=\min \left\{K u_{0}((s-t) / \sqrt{2}), 1\right\},
$$

then $\partial_{t} \bar{v}_{R, 0} \leq 0$.

Suppose that $\partial_{t} \bar{v}_{R, j-1} \leq 0$, we prove that $\partial_{t} \bar{v}_{R, j} \leq 0$. Indeed we have that $(m-1) / t^{2} \geq 0$. Moreover, for what said before, $\partial_{t} \bar{v}_{R, j} \leq 0$ on the lateral boundary of the set $T_{R} \times(0,+\infty)$ and it satisfies the Neumann condition

$$
-\partial_{\lambda}\left(\partial_{t} \bar{v}_{R, j}\right)+a \partial_{t} \bar{v}_{R, j}=g^{\prime}\left(\bar{v}_{R, j-1}\right) \partial_{t} \bar{v}_{R, j-1} \quad \text { on } T_{R} \times\{0\} .
$$

Assume by contradiction that $\partial_{t} \bar{v}_{R, j}$ is positive somewhere in $T_{R} \times \mathbb{R}^{+}$, then, by the maximum principle the $\sup \bar{v}_{R, j}>0$ will be achieved at some point $\left(x_{0}, 0\right)$ in $T_{R} \times\{0\}$. Since $g^{\prime}>0$ and $a>0$, applying Hopf's Lemma we get a contradiction with (5.10). This implies that $\partial_{t} \bar{v}_{R, j} \leq 0$ for every $j$ and then, passing to the limit, that $\partial_{t} \bar{v}_{R} \leq 0$.

Lemma 5.3. Let $\bar{u}_{R}$ be the function constructed in Lemma 5.1. Let $\bar{v}_{R}$ be the harmonic function in $T_{R} \times(0,+\infty)$ such that $\bar{v}_{R}(x, 0)=\bar{u}_{R}(x)$ for every $x \in T_{R}$ and $\bar{v}_{R}(x, \lambda)=u_{b}(x)$ for every $(x, \lambda) \in \partial T_{R} \times(0,+\infty)$.

Then, $\partial_{y} \bar{v}_{R} \geq 0$.

Proof. Consider as before the sequences of functions $\bar{v}_{R, j}$ and $\bar{u}_{R, j}$. We first observe that $\partial_{y} \bar{v}_{R, j} \geq 0$ on $\partial T_{R} \times(0,+\infty)$. Indeed $\bar{v}_{R, j} \equiv 0$ on the part of the boundary $\{t=s<R\} \times(0,+\infty)$. Thus, since $\partial_{y}$ is a tangential derivative here, we have $\partial_{y} \bar{v}_{R, j} \equiv 0$ on $\{t=s<R\} \times(0,+\infty)$.

Take now a point $(s=R, t, \lambda)$, with $0<t<R$, on the remaining part of the boundary. Recall that $\bar{v}_{R, j} \leq \bar{u}_{R, 0}=\min \left\{K u_{0}(z), 1\right\}=\min \left\{K u_{0}((s-t) / \sqrt{2}), 1\right\}$ in all of $T_{R} \times(0,+\infty)$.

Then, for every $0<\delta<t$ we have

$$
\begin{aligned}
\bar{v}_{R, j}(R-\delta, t-\delta, \lambda) & \leq \min \left\{K u_{0}\left(\frac{R-\delta-(t-\delta)}{\sqrt{2}}\right), 1\right\} \\
& =\min \left\{K u_{0}\left(\frac{R-t}{\sqrt{2}}\right), 1\right\}=u_{b}(R, t) .
\end{aligned}
$$

Then $\partial_{y} \bar{v}_{R, j} \geq 0$ on $\{t<s=R\} \times(0,+\infty)$.

Next, we consider the problem satisfied by $\partial_{t} \bar{v}_{R, j}$ and $\partial_{s} \bar{v}_{R, j}$. We recall that $\partial_{t} \bar{v}_{R, j}$ is a solution of (5.9) and $\partial_{s} \bar{v}_{R, j}$ satisfies

$$
\begin{cases}-\Delta\left(\partial_{s} \bar{v}_{R, j}\right)+\frac{(m-1)}{s^{2}} \partial_{s} \bar{v}_{R, j}=0 & \text { in } T_{R} \times(0, \infty) \\ -\partial_{\lambda}\left(\partial_{s} \bar{v}_{R, j}\right)+a \partial_{s} \bar{v}_{R, j}=g^{\prime}\left(\bar{v}_{R, j-1}\right) \partial_{s} \bar{v}_{R, j-1} & \text { on } T_{R} \times\{0\} .\end{cases}
$$


Thus, since $\partial_{y}=\left(\partial_{s}+\partial_{t}\right) / \sqrt{2}$, we have that $\partial_{y} \bar{v}_{R, j}$ satisfies the equation

$$
\begin{aligned}
-\Delta\left(\partial_{y} \bar{v}_{R, j}\right) & =-\frac{m-1}{\sqrt{2}}\left(\frac{\partial_{s} \bar{v}_{R, j}}{s^{2}}+\frac{\partial_{t} \bar{v}_{R, j}}{t^{2}}\right) \\
& =-\frac{m-1}{s^{2}} \partial_{y} \bar{v}_{R, j}-\frac{(m-1)\left(s^{2}-t^{2}\right)}{\sqrt{2} s^{2} t^{2}} \partial_{t} \bar{v}_{R, j} .
\end{aligned}
$$

Then $\partial_{y} \bar{v}_{R, j}$ is a solution of the problem

$$
\begin{cases}-\Delta\left(\partial_{y} \bar{v}_{R, j}\right)+\frac{(m-1)}{s^{2}} \partial_{y} \bar{v}_{R, j}+\frac{(m-1)\left(s^{2}-t^{2}\right)}{\sqrt{2} s^{2} t^{2}} \partial_{t} \bar{v}_{R, j}=0 \\ & \text { in } T_{R} \times(0, \infty) \\ \partial_{y} \bar{v}_{R, j} \geq 0 & \text { on } \partial T_{R} \times(0,+\infty) \\ -\partial_{\lambda}\left(\partial_{y} \bar{v}_{R, j}\right)+a \partial_{y} \bar{v}_{R, j}=g^{\prime}\left(\bar{v}_{R, j-1}\right) \partial_{y} \bar{v}_{R, j-1} & \text { on } T_{R} \times\{0\} .\end{cases}
$$

By the proof of Lemma 5.2 we see that $\partial_{t} \bar{v} \leq 0$ in $T_{R} \times(0,+\infty)$ and thus

$$
\frac{(m-1)\left(s^{2}-t^{2}\right)}{\sqrt{2} s^{2} t^{2}} \partial_{t} \bar{v}_{R, j} \leq 0, \quad \text { in } T_{R} \times(0,+\infty) .
$$

Then, we can apply, as in the proof of Lemma 5.2, the maximum principle and Hopf's Lemma, to obtain $\partial_{y} \bar{v}_{R, j} \geq 0$ for every $j$. Finally, passing to the limit for $j \rightarrow \infty$, we get $\partial_{y} \bar{v}_{R} \geq 0$ in $T_{R} \times(0,+\infty)$.

We can give now the proof of Proposition 1.7.

Proof of Proposition 1.7. In Lemma 5.1 we established the existence of a maximal solution $\bar{u}_{R}$ in $T_{R}$, that is, $\bar{u}_{R}$ is a solution of $D_{T_{R}, u_{b}} \bar{u}_{R}=f\left(\bar{u}_{R}\right)$ in $T_{R}$ and

$$
\bar{u}_{R} \geq u
$$

for every bounded solution $|u| \leq 1$ in $\mathbb{R}^{2 m}$ that vanishes on $\mathcal{C}$ and has the same sign as $s-t$.

By standard elliptic estimates and the compactness arguments as in the proof of Theorem 1.6, up to a subsequence we can take the limit as $R \rightarrow+\infty$ and obtain a solution $\bar{u}$ in $\mathcal{O}=\{s>t\}$, with $\bar{u}=0$ on $\mathcal{C}$. By construction,

$$
u \leq \bar{u}:=\lim _{R_{j} \rightarrow \infty} \bar{u}_{R_{j}},
$$

for all solutions $u$ as above. In addition, $\bar{u}$ depends only on $s$ and $t$.

By maximality of $\bar{u}$ and the existence of saddle solution of Theorem 1.6, we deduce that $\bar{u}>0$ in $\mathcal{O}$.

Since $f$ is odd, by odd reflection with respect to the Simons cone, we obtain a maximal solution $\bar{u}$ in $\mathbb{R}^{2 m}$ such that $|u| \leq|\bar{u}|$ in $\mathbb{R}^{2 m}$. 
Let $\bar{v}$ be the harmonic extension of $\bar{u}$ in $\mathbb{R}_{+}^{2 m+1}$. We prove now the monotonicity properties of $\bar{v}$.

By Lemmas 5.2 and 5.3, we have that $\partial_{t} \bar{v}_{R} \leq 0$ and $\partial_{y} \bar{v}_{R} \geq 0$ in $T_{R} \times(0,+\infty)$. Letting $R \rightarrow+\infty$, we get $\partial_{t} \bar{v} \leq 0$ and $\partial_{y} \bar{v} \geq 0$ in $\widetilde{\mathcal{O}}$. As a consequence $\partial_{s} \bar{v} \geq 0$ in $\widetilde{\mathcal{O}}$.

Since $v(s, t, \lambda)=-v(t, s, \lambda)$, it follows that $\partial_{s} \bar{v} \geq 0$ and $\partial_{t} \bar{v} \leq 0$ in $\mathbb{R}_{+}^{2 m+1}$.

Now, $\partial_{t} \bar{v} \leq 0$ in $\mathbb{R}_{+}^{2 m+1}$ and satisfies

$$
-\Delta \partial_{t} \bar{v}+\frac{m-1}{t^{2}} \partial_{t} \bar{v}=0 \quad \text { in } \mathbb{R}_{+}^{2 m+1}
$$

Then, the strong maximum principle implies that $\partial_{t} \bar{v}<0$ in $\mathbb{R}_{+}^{2 m+1} \backslash\{t=0\}$. Moreover we multiply by $t$ the following equation satisfied by $\bar{v}$ in $\mathbb{R}_{+}^{2 m+1}$

$$
\partial_{s s} \bar{v}+\partial_{t t} \bar{v}+\partial_{\lambda \lambda} \bar{v}+\frac{m-1}{s} \bar{v}_{s}+\frac{m-1}{t} \bar{v}_{t}=0
$$

Using that $\bar{v} \in C^{2}$ and letting $t \rightarrow 0$, we get $\partial_{t} \bar{v}=0$ on $\{t=0\}$. In the same way we deduce that $\partial_{s} \bar{v}>0$ in $\mathbb{R}_{+}^{2 m+1} \backslash\{s=0\}$ and $\partial_{s} \bar{v}=0$ on $\{s=0\}$. Recalling that $\partial_{z}=\left(\partial_{s}-\partial_{t}\right) / \sqrt{2}$, statement c) follows directly by a) and b). Finally, we remind that $\partial_{y} \bar{v}$ satisfies

$$
-\Delta \partial_{y} \bar{v}=-\frac{m-1}{s^{2}} \partial_{y} \bar{v}-\frac{(m-1)\left(s^{2}-t^{2}\right)}{\sqrt{2} s^{2} t^{2}} \partial_{t} \bar{v} \geq-\frac{m-1}{s^{2}} \partial_{y} \bar{v}
$$

in $\{s>t>0\} \times[0,+\infty)$, since $\partial_{t} \bar{v} \leq 0$ in this set. Since we have already proven that $\partial_{y} \bar{v} \geq 0$ in $\{s>t>0\} \times[0,+\infty)$, the strong maximum principle implies $\partial_{y} \bar{v}>0$ in $\{s>t>0\} \times[0,+\infty)$.

\section{Asymptotic behaviour of saddle solutions in $\mathbb{R}^{2 m}$}

In this section we study the asymptotic behaviour at infinity of solutions which are odd with respect to the Simons cone and positive in the set $\mathcal{O}=\{s>t\}$. In particular our result holds for saddle solutions.

We will consider the $(y, z)$ system of coordinates. Recall that we have defined in (1.17) $y$ and $z$ by

$$
\left\{\begin{array}{l}
y=\frac{s+t}{\sqrt{2}} \\
z=\frac{s-t}{\sqrt{2}}
\end{array}\right.
$$

which satisfy $y \geq 0$ and $-y \leq z \leq y$. 
We give the proof of Theorem 1.9, which states that any solution $u$ as above tends to infinity to the function

$$
U(x):=u_{0}(z)=u_{0}(d(x, \mathcal{C}))
$$

uniformly outside compact sets. We recall that $u_{0}$ is the layer solution of $(-\Delta)^{1 / 2} u_{0}=f\left(u_{0}\right)$ in $\mathbb{R}$ which vanishes at the origin, and $d(\cdot, \mathcal{C})$ denotes the distance to the Simons cone. Similarly $\nabla u$ converges to $\nabla U$. We will use this fact in the proof of instability of saddle solutions in dimension $2 m=4$ and $2 m=6$.

Our proof of the asymptotic behaviour follows a method used by Cabré and Terra for the classical equation $-\Delta u=f(u)$. They use a compactness argument based on translations of the solution, combined with two crucial Liouville-type results for nonlinear equations. Here, we use analog Liouville results for the nonlinear Neumann problem satisfied by the harmonic extension $v$ of our saddle solutions $u$. Both results were proven using the moving planes method.

The first result establishes a symmetry property for solutions of a nonlinear Neumann problem in the half-space, and it was proven in [18].

Theorem 6.1 ([18]). Let $\mathbb{R}_{+}^{n+1}=\left\{\left(x_{1}, x_{2}, \cdots, x_{n}, \lambda\right) \mid \lambda>0\right\}$ and let $f$ be such that $f(u) / u^{\frac{n+1}{n-1}}$ is non-increasing. Assume that $v$ is a solution of problem

$$
\begin{cases}-\Delta v=0 & \text { in } \mathbb{R}_{+}^{n+1}, \\ -\partial_{\lambda} v=f(v) & \text { on }\{\lambda=0\}, \\ v>0 & \text { in } \mathbb{R}_{+}^{n+1}\end{cases}
$$

Then $v$ depends only on $\lambda$.

More precisely, there exist $a \geq 0$ and $b>0$ such that

$$
v(x, \lambda)=v(\lambda)=a \lambda+b \text { and } f(b)=a .
$$

Remark 6.2. If $f$ satisfies hypothesis $(1.8),(1.9),(1.10)$, then $f(u) / u^{\frac{n+1}{n-1}}$ is nonincreasing.

Indeed, by Remark 3.4, $f(u) / u$ is non-increasing in $(0,1)$. Moreover, we can write

$$
\frac{f(u)}{u^{\frac{n+1}{n-1}}}=\frac{f(u)}{u} \cdot u^{1-\frac{n+1}{n-1}} .
$$

Since $(n+1) /(n-1)>1$, then $u^{1-\frac{n+1}{n-1}}$ is non-increasing, and thus $f$ satisfies the hypothesis of Theorem 6.1 above and Theorem 6.4 below.

Corollary 6.3. Let $f$ satisfy (1.8), (1.9), (1.10). Let $v$ be a bounded solution of problem (6.2).

Then, $v \equiv 0$ or $v \equiv 1$. 
Proof of Corollary 6.3. By Remark 6.2, $f$ satisfies the hypothesis of Theorem 6.1. Moreover since $f$ is bistable, we have that $f$ is odd, $f(0)=f( \pm 1)=0, f>0$ in $(0,1)$ and $f<0$ in $(1,+\infty)$. Then, since $v$ is bounded, necessarely we have $v(x, \lambda)=b$ with $f(b)=0$, that is $v \equiv 0$ or $v \equiv 1$.

The following theorem, proven in [9], establishes an analog symmetry property but for solutions in a quarter of space.

Theorem 6.4 ([9]). Let $\mathbb{R}_{++}^{n+1}=\left\{\left(x_{1}, x_{2}, \cdots, x_{n}, \lambda\right) \mid x_{n}>0, \lambda>0\right\}$ and let $f$ be such that $f(u) / u^{\frac{n+1}{n-1}}$ is non-increasing. Assume that $v$ is a bounded solution of problem

$$
\begin{cases}-\Delta v=0 & \text { in } \mathbb{R}_{++}^{n+1}, \\ -\partial_{\lambda} v=f(v) & \text { on }\left\{x_{n}>0, \lambda=0\right\} \\ v=0 & \text { on }\left\{x_{n}=0, \lambda \geq 0\right\} \\ v>0 & \text { in } \mathbb{R}_{++}^{n+1} .\end{cases}
$$

Then $v$ depends only on $x_{n}$ and $\lambda$.

Before proving Theorem 1.9, we give the following definition of semi-stability, which will be used in the proof of the asymptotic behaviour.

Definition 6.5. Let $\Omega \subset \mathbb{R}_{+}^{n+1}$ be an open set. Let $v$ be a bounded solution of

$$
\begin{cases}\Delta v=0 & \text { in } \Omega \\ -\partial_{\lambda} v=f(v) & \text { on } \partial^{0} \Omega .\end{cases}
$$

We say that $v$ is semi-stable in $\Omega$ if the second variation of the energy $\delta^{2} \mathcal{E} / \delta^{2} \xi^{2}$ with respect to perturbations $\xi$ with compact support in $\Omega \cup \partial^{0} \Omega$ is nonnegative.

That is, if

$$
Q_{v}(\xi)=\int_{\Omega}|\nabla \xi|^{2} d x d \lambda-\int_{\partial^{0} \Omega} f^{\prime}(u) \xi^{2} d x \geq 0,
$$

for all $\xi \in C_{c}^{\infty}\left(\Omega \cup \partial^{0} \Omega\right)$.

Now, we can give the proof of our asymptotic behaviour result.

Proof of Theorem 1.9. Let $u$ be a bounded solution of $(-\Delta)^{1 / 2} u=f(u)$ in $\mathbb{R}^{2 m}$ such that $u \equiv 0$ on $\mathcal{C}, u>0$ in $\mathcal{O}$, and $u$ is odd with respect to $\mathcal{C}$. Consider the harmonic extension $v$ of $u$ in $\mathbb{R}_{+}^{2 m+1}$, that satisfies

$$
\left\{\begin{array}{ll}
\Delta v=0 & \text { in } \mathbb{R}_{+}^{2 m+1} \\
-\partial_{\lambda} v=f(v) & \text { on } \partial \mathbb{R}_{+}^{2 m+1}
\end{array} .\right.
$$


Set $V(x, \lambda):=v_{0}(z, \lambda)$, where $v_{0}$ is the harmonic extension in $\mathbb{R}_{+}^{2}$ of the layer solution $u_{0}$ defined in (1.13). We want to prove that for every $\lambda \geq 0$

$$
v(x, \lambda)-V(x, \lambda) \rightarrow 0 \quad \text { and } \quad \nabla v(x, \lambda)-\nabla V(x, \lambda) \rightarrow 0,
$$

uniformly as $|x| \rightarrow \infty$.

Suppose that the theorem does not hold. Thus, there exists $\epsilon>0$ and a sequence $\left\{x_{k}\right\}$ with

$$
\left|x_{k}\right| \rightarrow \infty \quad \text { and } \quad\left|v\left(x_{k}, \lambda\right)-V\left(x_{k}, \lambda\right)\right|+\left|\nabla v\left(x_{k}, \lambda\right)-\nabla V\left(x_{k}, \lambda\right)\right| \geq \epsilon
$$

By continuity we may move slightly $x_{k}$ and assume $x_{k} \notin \mathcal{C}$ for all $k$. Moreover, up to a subsequence (which we still denote by $\left\{x_{k}\right\}$ ), either $\left\{x_{k}\right\} \subset\{s>t\}$ or $\left\{x_{k}\right\} \subset$ $\{s<t\}$. By the symmetries of the problem we may assume $\left\{x_{k}\right\} \subset\{s>t\}=\mathcal{O}$.

We distinguish two cases:

Case 1. $\left\{\operatorname{dist}\left(x_{k}, \mathcal{C}\right)=d_{k}\right\}$ is unbounded.

In this case, since $0<z_{k}=\operatorname{dist}\left(x_{k}, \mathcal{C}\right)=d_{k} \rightarrow+\infty$ (for a subsequence), we have that $V\left(x_{k}, \lambda\right)=v_{0}\left(z_{k}, \lambda\right)=v_{0}\left(d_{k}, \lambda\right)$ tends to 1 and $\left|\nabla V\left(x_{k}, \lambda\right)\right|$ tends to 0 , that is,

$$
V\left(x_{k}, \lambda\right) \rightarrow 1 \quad \text { and } \quad\left|\nabla V\left(x_{k}, \lambda\right)\right| \rightarrow 0 .
$$

From this and (6.4) we have

$$
\left|v\left(x_{k}, \lambda\right)-1\right|+\left|\nabla v\left(x_{k}, \lambda\right)\right| \geq \frac{\epsilon}{2},
$$

for $k$ large enough. Taking subsequence (and relabeling the subindex) we may assume $\operatorname{dist}\left(x_{k}, \mathcal{C}\right)=d_{k} \geq 2 k$.

Consider the ball $B_{k}(0) \subset \mathbb{R}^{2 m}$ of radius $k$ centered at $x=0$, and define

$$
w_{k}(\tilde{x}, \lambda)=v\left(\tilde{x}+x_{k}, \lambda\right) \text {, for every }(\tilde{x}, \lambda) \in B_{k}(0) \times(0,+\infty) .
$$

Since $B_{k}(0)+x_{k} \subset\{s>t\}$, we have that $0<w_{k}<1$ in $B_{k}(0) \times(0,+\infty)$ and

$$
\begin{cases}\Delta w_{k}=0 & \text { in } B_{k}(0) \times(0,+\infty) \\ -\partial_{\lambda} w_{k}=f(v) & \text { on } B_{k}(0) \times\{\lambda=0\} .\end{cases}
$$

Letting $k$ tend to infinity we obtain, through a subsequence, a nonnegative solution $w$ of the problem

$$
\begin{cases}-\Delta w=0 & \text { in } \mathbb{R}_{+}^{2 m+1} \\ -\partial_{\lambda} w=f(v) & \text { on } \partial \mathbb{R}_{+}^{2 m+1} \\ w>0 & \text { in } \mathbb{R}_{+}^{2 m+1} .\end{cases}
$$

Since $f$ satisfies (1.8), (1.9), (1.10), we have that, by Corollary $6.3, w \equiv 0$ or $w \equiv 1$. In either case, $\nabla w(0)=0$, that is, $\left|\nabla v\left(x_{k}, \lambda\right)\right|$ tends to 0 .

Next we show that $w \neq \equiv 0$. By Theorem 1.6 we have that $v$ is stable in $\mathcal{O} \times$ $(0,+\infty)$. Hence, $w_{k}$ is semi-stable in $B_{k}(0) \times(0,+\infty)\left(\right.$ since $\left.B_{k}(0)+x_{k} \subset \mathcal{O}\right)$ 
in the sense of Definition 6.5. This implies that $w$ is stable in all of $\mathbb{R}_{+}^{2 m+1}$ and therefore $w \not \equiv 0$ (otherwise, since $f^{\prime}(0)>0$ we could construct a test function $\xi$ such that $Q_{w}(\xi)<0$ which would be a contradiction with the fact that $w$ is stable).

Hence, it must be $w \equiv 1$. But this implies that $w(0, \lambda)=1$ and so $v\left(x_{k}, \lambda\right)$ tends to 1 . Therefore, we have that $v\left(x_{k}, \lambda\right)$ tends to 1 and $\left|\nabla v\left(x_{k}, \lambda\right)\right|$ tends to 0 , which is a contradiction with (6.5). We have proven the theorem in this Case 1.

Case 2. $\left\{\operatorname{dist}\left(x_{k}, \mathcal{C}\right)=d_{k}\right\}$ is bounded.

The points $x_{k}$ remain at a finite distance to the cone. Then, at least for a subsequence,

$$
d_{k} \rightarrow d \geq 0 \quad \text { as } k \rightarrow \infty .
$$

Let $x_{k}^{0} \in \mathcal{C}$ be a point that realizes the distance to the cone, that is,

$$
\operatorname{dist}\left(x_{k}, \mathcal{C}\right)=\left|x_{k}-x_{k}^{0}\right|=d_{k}
$$

and let $v_{k}^{0}$ be the inner unit normal to $\mathcal{C}=\partial \mathcal{O}$ at $x_{k}^{0}$. Note that $B_{d_{k}}\left(x_{k}\right) \subset \mathcal{O} \subset$ $\mathbb{R}^{2 m} \backslash \mathcal{C}$ and $x_{k}^{0} \in \partial B_{d_{k}}\left(x_{k}\right) \cap \mathcal{C}$, i.e., $x_{k}^{0}$ is the point where the sphere $\partial B_{d_{k}}\left(x_{k}\right)$ is tangent to the cone $\mathcal{C}$. It follows that $x_{k}^{0} \neq 0$ and that $\left(x_{k}-x_{k}^{0}\right) / d_{k}$ is the unit normal $v_{k}^{0}$ to $\mathcal{C}$ at $x_{k}^{0}$. That is, $x_{k}=x_{k}^{0}+d_{k} v_{k}^{0}$.

Now, since the sequence $\left\{v_{k}^{0}\right\}$ is bounded, there exists a subsequence such that

$$
v_{k}^{0} \rightarrow v \in \mathbb{R}^{2 m}, \quad|v|=1 .
$$

Write $w_{k}(\tilde{x}, \lambda)=v\left(\tilde{x}+x_{k}^{0}, \lambda\right)$, for $\tilde{x} \in \mathbb{R}^{2 m}$. The functions $w_{k}$ are all solutions of

$$
\begin{cases}\Delta w_{k}=0 & \text { in } \mathbb{R}_{+}^{2 m+1} \\ -\partial_{\lambda} w_{k}=f\left(w_{k}\right) & \text { on } \partial \mathbb{R}_{+}^{2 m+1},\end{cases}
$$

and are uniformly bounded. Hence, by elliptic estimates, the sequence $\left\{w_{k}\right\}$ converges locally in space in $C^{2}$, up to a subsequence, to a solution $w$ in $\mathbb{R}_{+}^{2 m+1}$. Therefore we have that, as $k$ tends to infinity and up to a subsequence,

$$
w_{k} \rightarrow w \text { and } \nabla w_{k} \rightarrow \nabla w \text { uniformly on compact sets of } \mathbb{R}_{+}^{2 m+1},
$$

where $w$ is a solution

$$
\begin{cases}\Delta w=0 & \text { in } \mathbb{R}_{+}^{2 m+1} \\ -\partial_{\lambda} w=f(w) & \text { on } \partial \mathbb{R}_{+}^{2 m+1}\end{cases}
$$

Note that the curvature of $\mathcal{C}$ at $x_{k}^{0}$ goes to zero as $k$ tends to infinity, since $\mathcal{C}$ is a cone and $\left|x_{k}\right| \rightarrow \infty$ (note that $\left|x_{k}^{0}\right| \rightarrow \infty$ due to $\left|x_{k}\right| \rightarrow \infty$ and $\left|x_{k}-x_{k}^{0}\right|=d_{k} \rightarrow$ 
$d<\infty)$. Thus, $\mathcal{C}$ at $x_{k}^{0}$ is flatter and flatter as $k \rightarrow \infty$ and since we translate $x_{k}^{0}$ to 0 , the limiting solution $w$ satisfies

$$
\begin{cases}\Delta w=0 & \text { in } M:=\left\{(x, \lambda) \in \mathbb{R}_{+}^{2 m+1}: \tilde{x} \cdot v=0, \lambda>0\right\} \\ w \geq 0 & \text { in } M \\ w=0 & \text { on }\{\tilde{x} \cdot v=0\} \\ -\partial_{\lambda} w=f(w) & \text { on }\{\lambda=0\} .\end{cases}
$$

For the details of the proof of this fact see [11].

Now, since $v$ is stable for perturbations vanishing on $\partial \mathcal{O} \times \mathbb{R}^{+}$, it follows that $w$ is stable for perturbations with compact support in $M$, and therefore $w$ can not be identically zero. By Theorem 6.4, since $f$ satisfies (1.8), (1.9), (1.10), we deduce that $w$ is symmetric, that is, it is a function of only two variable (the orthogonal direction to $H$ and $\lambda$ ). It follows that

$$
w(\tilde{x}, \lambda)=v_{0}(\tilde{x} \cdot \nu, \lambda) \quad \text { for all }(\tilde{x}, \lambda) \in M .
$$

From the definition of $w_{k}$, and using that $z_{k}=d_{k}=\left|x_{k}-x_{k}^{0}\right|$ is a bounded sequence and that $x_{k}-x_{k}^{0}=d_{k} v_{k}^{0}$, we have that

$$
\begin{aligned}
v\left(x_{k}, \lambda\right) & =w_{k}\left(x_{k}-x_{k}^{0}, \lambda\right)=w\left(x_{k}-x_{k}^{0}, \lambda\right)+\mathrm{o}(1)=v_{0}\left(\left(x_{k}-x_{k}^{0}\right) \cdot v, \lambda\right)+\mathrm{o}(1) \\
& =v_{0}\left(\left(x_{k}-x_{k}^{0}\right) \cdot v_{k}^{0}, \lambda\right)+\mathrm{o}(1)=v_{0}\left(d_{k}, \lambda\right)+\mathrm{o}(1) \\
& =v_{0}\left(z_{k}, \lambda\right)+\mathrm{o}(1)=V\left(x_{k}, \lambda\right)+\mathrm{o}(1) .
\end{aligned}
$$

The same argument can be done for $\nabla v\left(x_{k}, \lambda\right)$ and $\nabla V\left(x_{k}, \lambda\right)$. We arrive to a contradiction with (6.4).

\section{Instability in dimensions 4 and 6}

Before proving the theorem on the instability of saddle solutions in dimensions 4 and 6 , we establish a lemma that will be useful later.

Lemma 7.1. Assume that $f$ satisfies conditions (1.8), (1.9), (1.10). Let $v$ be a bounded solution of (1.3) in $\mathbb{R}_{+}^{n+1}$ and $w$ a function such that $|v| \leq|w| \leq 1$ in $\mathbb{R}_{+}^{n+1}$. Then,

$$
Q_{v}(\xi) \leq Q_{w}(\xi) \quad \text { for all } \xi \in C_{0}^{\infty}\left(\overline{\mathbb{R}_{+}^{n+1}}\right)
$$

where $Q_{w}$ is defined by

$$
Q_{w}(\xi)=\int_{\mathbb{R}_{+}^{n+1}}|\nabla \xi|^{2} d x d \lambda-\int_{\partial \mathbb{R}_{+}^{n+1}} f^{\prime}(w) \xi^{2} d x
$$

In particular, if there exists a function $\xi \in C_{0}^{\infty}\left(\overline{\mathbb{R}_{+}^{n+1}}\right)$ such that $Q_{w}(\xi)<0$, then $v$ is unstable. 
Proof. Let $v$ be a bounded solution of (1.3) and $w$ a function with $|v| \leq|w| \leq 1$.

Since $f^{\prime}$ is decreasing in $(0,1)$ we have that

$$
f^{\prime}(|v|) \geq f^{\prime}(|w|) \quad \text { in } \mathbb{R}_{+}^{n+1} .
$$

Moreover, $f^{\prime}$ being even yields,

$$
f^{\prime}(v) \geq f^{\prime}(w) \quad \text { in } \mathbb{R}_{+}^{n+1},
$$

so that

$$
Q_{v}(\xi) \leq Q_{w}(\xi),
$$

for every test function $\xi \in C_{0}^{\infty}\left(\overline{\mathbb{R}_{+}^{n+1}}\right)$.

Hence, if there exists $\xi_{0}$ such that $Q_{w}\left(\xi_{0}\right)<0$, then also $Q_{v}\left(\xi_{0}\right)<0$. That is, $v$ is unstable.

In the proof of the instability results for dimension 4 and 6 we use the maximal solution $\bar{v}$ of problem (1.3) and, more importantly, the equation satisfied by $\bar{v}_{z}=\partial_{z} \bar{v}$. We prove that this solution $\bar{v}$ is unstable by constructing a test function $\xi(y, z, \lambda)=\eta(y, \lambda) \bar{v}_{z}(y, z, \lambda)$ such that $Q_{\bar{v}}(\xi)<0$. Two crucial ingredients will be the asymptotic behaviour and monotonicity results for $\bar{v}$ (Theorems 1.9 and 1.7). Since $\bar{v}$ is maximal, Lemma 7.1 implies that all bounded solutions $-1 \leq v \leq 1$ vanishing on $\mathcal{C} \times \mathbb{R}^{+}$and having the same sign as $s-t$ are also unstable.

We recall that if $v$ is a function depending only on $s, t$ and $\lambda$, then the second variation of the energy is given by

$$
\begin{aligned}
c_{m} Q_{v}(\xi)= & \int_{0}^{+\infty} \int_{\{s>0, t>0\}} s^{m-1} t^{m-1}\left(\xi_{s}^{2}+\xi_{t}^{2}+\xi_{\lambda}^{2}\right) d s d t d \lambda \\
& -\int_{\{s>0, t>0\}} s^{m-1} t^{m-1} f^{\prime}(v) \xi^{2} d s d t
\end{aligned}
$$

where $c_{m}$ is a positive constant depending on $m$. Here, the perturbations are of the form $\xi=\xi(s, t, \lambda)$ and vanishes for $s, t$ and $\lambda$ large enough.

Moreover, if we change to variables $(y, z, \lambda)$, for a different constant $c_{m}$ we get,

$$
\begin{aligned}
c_{m} Q_{v}(\xi)= & \int_{0}^{+\infty} \int_{\{-y<z<y\}}\left(y^{2}-z^{2}\right)^{m-1}\left(\xi_{y}^{2}+\xi_{z}^{2}+\xi_{\lambda}^{2}\right) d y d z d \lambda \\
& -\int_{\{-y<z<y\}}\left(y^{2}-z^{2}\right)^{m-1} f^{\prime}(v) \xi^{2} d y d z,
\end{aligned}
$$

where $\xi=\xi(y, z, \lambda)$ vanishes for $y$ and $\lambda$ large enough. 
Proof of Theorem 1.10. We begin by establishing that the maximal solution $\bar{v}$ is unstable in dimension $2 m=4$ and $2 m=6$. As said before, using that $\bar{v}$ is maximal and applying Lemma 7.1, we deduce the instability of $v$ in dimensions 4 and 6 .

We have, for every test function $\xi$,

$$
Q_{\bar{v}}(\xi)=\int_{\mathbb{R}_{+}^{2 m+1}}|\nabla \xi|^{2} d x d \lambda-\int_{\partial \mathbb{R}_{+}^{2 m+1}} f^{\prime}(\bar{v}) \xi^{2} d x
$$

Suppose now that $\xi=\xi(y, z, \lambda)=\eta(y, z, \lambda) \psi(y, z, \lambda)$. For $\xi$ to be Lipschitz and of compact support in $\overline{\mathbb{R}_{+}^{2 m+1}}$, we need $\eta$ and $\psi$ to be Lipschitz functions of compact support in $y \in[0,+\infty)$ and $\lambda \in[0,+\infty)$. The expression for $Q_{\bar{v}}$ becomes,

$$
\begin{aligned}
Q_{\bar{v}}(\xi)=\int_{0}^{+\infty} & \int_{\mathbb{R}^{2 m}}\left(|\nabla \eta|^{2} \psi^{2}+\eta^{2}|\nabla \psi|^{2}+2 \eta \psi \nabla \eta \cdot \nabla \psi\right) d x d \lambda \\
& -\int_{\mathbb{R}^{2 m}} f^{\prime}(\bar{v}) \eta^{2} \psi^{2} d x
\end{aligned}
$$

Using that $2 \eta \psi \nabla \eta \cdot \nabla \psi=\psi \nabla\left(\eta^{2}\right) \cdot \nabla \psi$, and integrating by parts this term we have

$$
\begin{aligned}
Q_{\bar{v}}(\xi)= & \int_{0}^{+\infty} \int_{\mathbb{R}^{2 m}}\left(|\nabla \eta|^{2} \psi^{2}-\eta^{2} \psi \Delta \psi\right) d x d \lambda \\
& -\int_{\mathbb{R}^{2 m}}\left(\psi(y, z, 0) \eta^{2} \partial_{\lambda} \psi(y, z, 0)+f^{\prime}(\bar{v}) \eta^{2} \psi^{2}\right) d x
\end{aligned}
$$

that is,

$$
Q_{\bar{v}}(\xi)=\int_{0}^{+\infty} \int_{\mathbb{R}^{2 m}}\left(|\nabla \eta|^{2} \psi^{2}-\eta^{2} \psi \Delta \psi\right) d x d \lambda-\int_{\mathbb{R}^{2 m}} \eta^{2} \psi\left(\partial_{\lambda} \psi+f^{\prime}(\bar{v}) \psi\right) d x
$$

Choose $\psi(y, z, \lambda)=\bar{v}_{z}(y, z, \lambda)$. We consider now problem (1.3), which is satisfied by $\bar{v}$, written in the $(y, z, \lambda)$ variables

$$
\begin{cases}\bar{v}_{y y}+\bar{v}_{z z}+\bar{v}_{\lambda \lambda}+\frac{2(m-1)}{y^{2}-z^{2}}\left(y \bar{v}_{y}-z \bar{v}_{z}\right)=0 & \text { in } \mathbb{R}_{+}^{2 m+1} \\ -\partial_{\lambda} \bar{v}=f(\bar{v}) & \text { on } \partial \mathbb{R}_{+}^{2 m+1} .\end{cases}
$$

If we differentiate these equations written in $(y, z, \lambda)$ variables with respect to $z$, we find

$$
\begin{cases}\Delta \bar{v}_{z}-\frac{2(m-1)}{y^{2}-z^{2}} \bar{v}_{z}+\frac{4(m-1) z}{\left(y^{2}-z^{2}\right)^{2}}\left(y \bar{v}_{y}-z \bar{v}_{z}\right)=0 & \text { in } \mathbb{R}_{+}^{2 m+1} \\ -\partial_{\lambda} \bar{v}_{z}=f^{\prime}(\bar{v}) \bar{v}_{z} & \text { on } \partial \mathbb{R}_{+}^{2 m+1} .\end{cases}
$$


Replacing in the expression for $Q_{\bar{v}}$ we obtain,

$$
\begin{aligned}
Q_{\bar{v}}(\xi)= & \int_{0}^{+\infty} \int_{\mathbb{R}^{2 m}}\left(|\nabla \eta|^{2} \bar{v}_{z}^{2}\right. \\
& \left.-\eta^{2}\left\{\frac{2(m-1)\left(y^{2}+z^{2}\right)}{\left(y^{2}-z^{2}\right)^{2}} \bar{v}_{z}^{2}-\frac{4(m-1) z y}{\left(y^{2}-z^{2}\right)^{2}} \bar{v}_{y} \bar{v}_{z}\right\}\right) d x d \lambda
\end{aligned}
$$

Next we change coordinates to $(y, z, \lambda)$ and we have, for some positive constant $c_{m}$,

$$
\begin{aligned}
c_{m} Q_{\bar{v}}(\xi)= & \int_{0}^{+\infty} \int_{\{-y<z<y\}}\left(y^{2}-z^{2}\right)^{m-1}\left(|\nabla \eta|^{2} \bar{v}_{z}^{2}\right. \\
& \left.-\eta^{2}\left\{\frac{2(m-1)\left(y^{2}+z^{2}\right)}{\left(y^{2}-z^{2}\right)^{2}} \bar{v}_{z}^{2}-\frac{4(m-1) z y}{\left(y^{2}-z^{2}\right)^{2}} \bar{v}_{y} \bar{v}_{z}\right\}\right) d y d z d \lambda .
\end{aligned}
$$

Now choose $\eta(y, z, \lambda)=\eta_{1}(y) \eta_{2}(\lambda)$, where $\eta_{1}$ and $\eta_{2}$ are smooth functions with compact support in $[0,+\infty)$. Moreover we require that $\eta_{2}(\lambda) \equiv 1$ for $\lambda<N$ and $\eta_{2}(\lambda) \equiv 0$ for $\lambda>N+1$, where $N$ is a large positive number that we will choose later. For $a>1$, a constant that we will make tend to infinity, let $\phi=\phi(\rho)$ be a Lipschitz function of $\rho:=y / a$ with compact support $\left[\rho_{1}, \rho_{2}\right] \subset[0,+\infty)$. Let us denote by

$$
\begin{gathered}
\eta_{1}^{a}(y):=\phi(y / a) \quad \text { and } \\
\xi_{a}(y, z, \lambda)=\eta_{1}^{a}(y) \eta_{2}(\lambda) \bar{v}_{z}(y, z, \lambda)=\phi(y / a) \eta_{2}(\lambda) \bar{v}_{z}(y, z, \lambda) .
\end{gathered}
$$

The change $y=a \rho, d y=a d \rho$ yields,

$$
\begin{aligned}
& c_{m} Q_{\bar{v}}\left(\xi_{a}\right) \\
& =a^{2 m-3} \int_{0}^{N+1} \int_{\rho_{1}}^{\rho_{2}} \int_{\{-a \rho<z<a \rho\}} \rho^{2(m-1)}\left(1-\frac{z^{2}}{a^{2} \rho^{2}}\right)^{m-1}\left(\phi_{\rho}^{2} \eta_{2}^{2}(\lambda) \bar{v}_{z}^{2}\right. \\
& +a^{2} \phi^{2}(\rho)\left(\eta_{2}^{\prime}\right)^{2} \bar{v}_{z}^{2} \\
& \left.-\phi^{2} \eta_{2}^{2}\left\{\frac{2(m-1)\left(1+\frac{z^{2}}{a^{2} \rho^{2}}\right)}{\rho^{2}\left(1-\frac{z^{2}}{a^{2} \rho^{2}}\right)^{2}} \bar{v}_{z}^{2}-\frac{4(m-1) z}{a \rho^{3}\left(1-\frac{z^{2}}{a^{2} \rho^{2}}\right)^{2}} \bar{v}_{y} \bar{v}_{z}\right\}\right) d z d \rho d \lambda
\end{aligned}
$$


Dividing by $a^{2 m-3} N$ and using that $\left(1-\frac{z^{2}}{a^{2} \rho^{2}}\right)^{2} \leq 1$ and $1+\frac{z^{2}}{a^{2} \rho^{2}} \geq 1$, we obtain

$$
\begin{aligned}
& \frac{c_{m} Q_{\bar{u}}\left(\xi_{a}\right)}{a^{2 m-3} N} \\
& \leq \frac{1}{N} \int_{0}^{N+1} \int_{\rho_{1}}^{\rho_{2}} \int_{\{-a \rho<z<a \rho\}} \rho^{2(m-1)} \eta_{2}^{2} \bar{v}_{z}^{2}(a \rho, z, \lambda)\left(\phi_{\rho}^{2}-\frac{2(m-1)}{\rho^{2}} \phi^{2}\right) d z d \rho d \lambda \\
& \quad+\frac{a^{2}}{N} \int_{N}^{N+1} \int_{\rho_{1}}^{\rho_{2}} \int_{\{-a \rho<z<a \rho\}} \rho^{2(m-1)} \phi^{2}\left(\eta_{2}^{\prime}\right)^{2} \bar{v}_{z}^{2} d z d \rho d \lambda \\
& \quad+\frac{1}{N} \int_{0}^{N+1} \int_{\rho_{1}}^{\rho_{2}} \int_{\{-a \rho<z<a \rho\}} \frac{4(m-1) z \rho^{2 m-5} \eta_{2}^{2} \phi^{2}(\rho)}{a} \bar{v}_{y}(a \rho, z, \lambda) \bar{v}_{z}(a \rho, z, \lambda) d z d \rho d \lambda . \\
& =I_{1}+I_{2}+I_{3} .
\end{aligned}
$$

We study these three integrals separately.

Consider first $I_{3}$. From Theorem 1.9 we have that $\bar{v}_{y}(a \rho, z, \lambda) \rightarrow 0$ uniformly, for all $\rho \in\left[\rho_{1}, \rho_{2}\right]=\operatorname{supp} \phi$, as $a$ tends to infinity. Hence, given $\epsilon>0$, for $a$ sufficiently large, $\left|\bar{v}_{y}(a \rho, z)\right| \leq \epsilon$. Moreover, we have seen in Theorem 1.7 that $\bar{v}_{z} \geq 0$. Hence, since $\phi$ is bounded, for $a$ large we have

$$
\begin{aligned}
I_{3} & \leq\left|\frac{1}{N} \int_{0}^{N+1} \eta_{2}^{2} \int \frac{4(m-1) z \rho^{2 m-5} \phi^{2}(\rho)}{a} \bar{v}_{y} \bar{v}_{z} d \rho d z d \lambda\right| \\
& \leq \frac{1}{N} \int_{0}^{N+1} \eta_{2}^{2} \int\left|\frac{4(m-1) z \rho^{2 m-5} \phi^{2}(\rho)}{a}\right| \bar{v}_{y} \mid \bar{v}_{z} d \rho d z d \lambda \\
& \leq \frac{1}{N} \int_{0}^{N+1} \eta_{2}^{2} \int 4(m-1) \rho^{2 m-4} \phi^{2}(\rho)\left|\bar{v}_{y}\right| \bar{v}_{z} d \rho d z d \lambda \\
& \leq \frac{C \epsilon}{N} \int_{\rho_{1}}^{\rho_{2}} \rho^{2 m-4} d \rho \int_{0}^{N+1} \eta_{2}^{2} d \lambda \int_{-a \rho}^{a \rho} \bar{v}_{z} d z \\
& =\frac{C \epsilon}{N} \int_{0}^{N+1} \eta_{2}^{2} \int_{\rho_{1}}^{\rho_{2}}(\bar{v}(a \rho, a \rho, \lambda)-\bar{v}(a \rho,-a \rho, \lambda)) d \rho d \lambda \\
& \leq C \epsilon
\end{aligned}
$$

where $C$ are different constants depending on $\rho_{1}$ and $\rho_{2}$. Hence, as $a$ tends to infinity, this integral converges to zero. 
Now, consider $I_{2}$ and choose $N$ such that $a^{2} / N \leq 1 / a^{2}$. With this choice of $N$, we have

$$
\begin{aligned}
I_{2} & =\frac{a^{2}}{N} \int_{N}^{N+1} \int_{\rho_{1}}^{\rho_{2}} \int_{\{-a \rho<z<a \rho\}} \rho^{2(m-1)} \phi^{2}\left(\eta_{2}^{\prime}\right)^{2} \bar{v}_{z}^{2} \\
& \leq \frac{1}{a^{2}} \int_{N}^{N+1} \int_{\rho_{1}}^{\rho_{2}} \int_{\{-a \rho<z<a \rho\}} \rho^{2(m-1)} \phi^{2}\left(\eta_{2}^{\prime}\right)^{2} \bar{v}_{z}^{2} \\
& \leq \frac{C}{a} \sup \bar{v}_{z}^{2} .
\end{aligned}
$$

Thus, $I_{2}$ tends to 0 as $a \rightarrow \infty$.

Next, consider $I_{1}$. We have that, again by Theorem $1.9, \bar{v}_{z}(a \rho, z, \lambda)$ converges to $\partial_{z} v_{0}(z, \lambda)$ which is a bounded positive integrable function. We write

$$
\begin{aligned}
I_{1}= & \frac{1}{N} \int_{0}^{N+1} \eta_{2}^{2} \int_{\rho_{1}}^{\rho_{2}} \int_{\{-a \rho<z<a \rho\}} \rho^{2(m-1)} \bar{v}_{z}^{2}(a \rho, z, \lambda)\left(\phi_{\rho}^{2}-\frac{2(m-1)}{\rho^{2}} \phi^{2}\right) d \rho d z d \lambda \\
= & \frac{1}{N} \int_{0}^{N+1} \eta_{2}^{2} \int_{\rho_{1}}^{\rho_{2}} \int_{\{-a \rho<z<a \rho\}}\left(\partial_{z} v_{0}\right)^{2} \rho^{2(m-1)}\left(\phi_{\rho}^{2}-\frac{2(m-1)}{\rho^{2}} \phi^{2}\right) d \rho d z d \lambda \\
& +\frac{1}{N} \int_{0}^{N+1} \eta_{2}^{2} \int_{\rho_{1}}^{\rho_{2}} \int_{\{-a \rho<z<a \rho\}} \rho^{2(m-1)}\left(\bar{v}_{z}(a \rho, z, \lambda)-\partial_{z} v_{0}(z, \lambda)\right)\left(\bar{v}_{z}(a \rho, z, \lambda)\right. \\
& \left.+\partial_{z} v_{0}(z, \lambda)\right)\left(\phi_{\rho}^{2}-\frac{2(m-1)}{\rho^{2}} \phi^{2}\right) d \rho d z d \lambda .
\end{aligned}
$$

For $a$ large, $\left|\bar{v}_{z}(a \rho, z, \lambda)-\partial_{z} v_{0}(z, \lambda)\right| \leq \epsilon$ in $\left[\rho_{1}, \rho_{2}\right]$. In addition $\bar{v}_{z}(a \rho, z, \lambda)+$ $\partial_{z} v_{0}(z, \lambda)$ is positive and is a derivative with respect to $z$ of a bounded function, thus it is integrable in $z$. Hence, since $\phi=\phi(\rho)$ is smooth with compact support, the second integral converges to zero as $a$ tends to infinity. Therefore, letting $a$ tend to infinity, we obtain

$$
\begin{aligned}
& \limsup _{a \rightarrow \infty} \frac{c_{m} Q_{\bar{v}}\left(\xi_{a}\right)}{a^{2 m-3} N} \\
& \leq \limsup _{a \rightarrow \infty} \frac{1}{N}\left(\int_{0}^{N+1} d \lambda \eta_{2}^{2} \int_{0}^{+\infty} d z\left(\partial_{z} v_{0}\right)^{2}(z)\right) \\
& \quad \cdot \int d \rho \rho^{2(m-1)}\left(\phi_{\rho}^{2}-\frac{2(m-1)}{\rho^{2}} \phi^{2}\right) \\
& \leq C \int_{0}^{+\infty}\left(\partial_{z} v_{0}\right)^{2}(z) d z \int \rho^{2(m-1)}\left(\phi_{\rho}^{2}-\frac{2(m-1)}{\rho^{2}} \phi^{2}\right) d \rho .
\end{aligned}
$$

Finally, we prove that when $2 m=4$ and $2 m=6$, there exists a test function $\phi$ for which

$$
\int \rho^{2(m-1)}\left(\phi_{\rho}^{2}-\frac{2(m-1)}{\rho^{2}} \phi^{2}\right) d \rho<0 .
$$


The integral in $\rho$ can be seen as an integral in $\mathbb{R}^{2 m-1}$ of radial functions $\phi=$ $\phi(|x|)=\phi(\rho)$.

Hardy's inequality in $\mathbb{R}^{2 m-1}$ states (see $e . g .[15]$ ) that

$$
\frac{(2 m-1-2)^{2}}{4} \int_{\mathbb{R}^{2 m-1}} \frac{\varphi^{2}}{|x|^{2}} d x \leq \int_{\mathbb{R}^{2 m-1}}|\nabla \varphi|^{2} d x
$$

holds for every $H^{1}$ function $\varphi$ with compact support, and the constant $(2 m-1-$ $2)^{2} / 4$ is the best possible. Using this inequality we have that the integral in (7.5) is positive for all Lipschitz $\phi$ with compact support if and only if

$$
2(m-1) \leq \frac{(2 m-1-2)^{2}}{4} .
$$

Writing $n=2 m$, the above inequality holds if and only if

$$
n^{2}-10 n+17 \geq 0
$$

that is, $n \geq 8$. Thus, when $2 m=4$ and $2 m=6$, we have that the integral (7.5) is negative for some compactly supported Lipschitz function $\phi=\phi(\rho)$ and then we conclude that the limsup in (7.4) is negative for such $\phi$ and hence that $\bar{u}$ is unstable.

Remark 7.2. We observe that for $n \geq 8$ the limsup in (7.4) is nonnegative for every $\phi$ and we conclude a certain asymptotic stability at infinity of $\bar{v}$.

\section{References}

[1] G. Alberti, L. Ambrosio and X. CABré, On a long-standing conjecture of E. De Giorgi: symmetry in $3 D$ for general nonlinearities and a local minimality property, Acta Appl. Math. 65 (2001), 9-33.

[2] L. AMBrosio and X. CABRÉ, Entire solutions of semilinear elliptic equations in $\mathbb{R}^{3}$ and a Conjecture of De Giorgi, J. Amer. Math. Soc. 13 (2000), 725-739.

[3] E. Bombieri, E. De GiORGi and E. Giusti, Minimal cones and the Bernstein problem, Invent. Math. 7 (1969), 243-268.

[4] X. CABRÉ and E. CINTI, Energy estimates and 1-D symmetry for nonlinear equations involving the half-Laplacian, Discrete Contin. Dyn. Syst. 28 (2010), 1179-1206. A special issue dedicated to Louis Nirenberg on the Occasion of his 85th Birthday.

[5] X. CABRÉ and E. CINTI, Sharp energy estimates for nonlinear fractional diffusion equations, Calculus of Variations and Partial Differential Equations, to appear (DOI: 10.1007/s00526-012-0580-6).

[6] X. CABRÉ and Y. SIRE, Nonlinear equations for fractional Laplacians I: regularity, maximum principles, and Hamiltonian estimates, accepted for publication in Trans. Amer. Mat. Soc.

[7] X. CABré and Y. SIRE, Nonlinear equations for fractional Laplacians II: existence, uniqueness, and qualitative properties of solutions, forthcoming.

[8] X. CABRÉ and J. SOLÀ-MORALES, Layer solutions in a halph-space for boundary reactions, Comm. Pure and Appl. Math. 58 (2005), 1678-1732. 
[9] X. CABRÉ and J. TAN, Positive solutions of nonlinear problems involving the square root of the Laplacian, Adv. Math. 224 (2010), 2052-2093.

[10] X. CABRÉ and J. TERRA, Saddle-shaped solutions of bistable diffusion equations in all of $\mathbb{R}^{2 m}$, J. Eur. Math. Soc. 11 (2009), 819-843.

[11] X. CABRÉ and J. TERRA, Qualitative properties of saddle-shaped solutions to bistable diffusion equations, Comm. Partial Differential Equations 35 (2010), 1923-1957.

[12] L. Caffarelli and L. Silvestre, An estension related to the fractional Laplacian, Comm. Partial Differential Equations 32 (2007), 1245-1260.

[13] H. DANG, P. C. FifE and L. A. Peletier, Saddle solutions of the bistable diffusion equation, Z. Angew Math. Phys. 43 (1992), 984-998.

[14] M. Del Pino, M. KowALCZYK and J. WeI, On De Giorgi conjecture in dimension $N \geq 9$, Ann. of Math. 174 (2011), 1485-1569.

[15] J. P. Garcia Azorero and I. Peral, Hardy inequalities and some critical elliptic and parabolic problems, J. Differential Equations 144 (1998), 441-476.

[16] N. Ghoussoub and C. Gui, On a conjecture of De Giorgi and some related problems, Math. Ann. 311 (1998), 481-491.

[17] D. JERISON and R. MONNEAU, Towards a counter-example to a conjecture of De Giorgi in high dimensions, Ann. Mat. Pura Appl. 183 (2004), 439-467.

[18] Y. Y. LI and L. ZHANG, Liouville-type theorems and Harnack-type inequalities for semilinear elliptic equations, J. Anal. Math. 90 (2003), 27-87.

[19] L. ModicA, A gradient bound and a Liouville theorem for nonlinear Poisson equations, Comm. Pure Appl. Math. 38 (1985), 679-684.

[20] O. S AVIN, Phase transitions: regularity of flat level sets, Ann. of Math. 169 (2009), 41-78.

[21] M. SChATZMAn, On the stability of the saddle solution of Allen-Cahn's equation, Proc. Roy. Soc. Edinburgh Sect. A 125 (1995), 1241-1275.

[22] Y. SIRE and E. VALDINOCI, Fractional Laplacian phase transitions and boundary reactions: A geometric inequality and a symmetry result, J. Funct. Anal. 256 (2009), 1842-1864.

[23] J. F. Toland, The Peierls-Nabarro and Benjamin-Ono equations, J. Funct. Anal. 145 (1997), 136-150.

Dipartimento di Matematica Università degli Studi di Bologna Piazza di Porta S. Donato, 5 40126 Bologna, Italia eleonora.cinti5@unibo.it 